\title{
Azavinyl Azomethine Ylides from Thermal Ring Opening of a-Aziridinohydrazones: Unprecedented 1,5-Electrocyclization to Imidazoles
}

Orazio A. Attanasi, ${ }^{*} \dagger$ Paolo Davoli, ${ }^{\ddagger}$ Gianfranco Favi, ${ }^{\dagger}$ Paolino Filippone, ${ }^{\dagger}$ Arrigo Forni, ${ }^{\ddagger}$ Giada Moscatelli, ${ }^{\dagger}$ and Fabio Prati*, ${ }^{\dagger}$

Istituto di Chimica Organica della Facoltà di Scienze e Tecnologie, Università degli Studi di Urbino "Carlo Bo", Via I Maggetti 24, 61029 Urbino, Italy and Dipartimento di Chimica, Università di Modena e Reggio Emilia, Via Campi 183, 41100 Modena, Italy

E-mail:attanasi@uniurb.it; prati.fabio@unimore.it

${ }^{\dagger}$ Università di Urbino

¥ Università di Modena e Reggio Emilia

\section{SUPPORTING INFORMATION}

Table of Contents

1. General remarks

2. Experimental procedures and spectral data

trans-3-Phenyl-2-aziridinecarboxylic acid methyl ester (2a)

2,2-Aziridinedicarboxylic acid dimethyl ester (2b)

Synthesis of aziridinecarboxylate 2c

2-Phenyl-2-oxiranecarboxylic acid methyl ester (2f)

3-Azido-2-hydroxy-2-phenylpropionic acid methyl ester (2g)

2-Phenyl-2-aziridinecarboxylic acid methyl ester (2c)

Synthesis of $\alpha$-aziridinohydrazones 3a-k

Synthesis of imidazoles $\mathbf{4 a - k}$

3. ${ }^{1} \mathrm{H}$ and ${ }^{13} \mathrm{C}$ NMR spectra of all imidazoles 


\section{General Remarks.}

All reactions requiring anhydrous conditions were carried out using oven-dried glassware. Commercially grade reagents and solvents were used without further purification; methanol and acetonitrile were dried by storage upon $3 \AA$ molecular sieves. 1,2-Diaza-1,3-butadienes 1a-e were synthesized as a mixture of $E / Z$ isomers as already described. ${ }^{1,2}$ Aziridinecarboxylates $\mathbf{2} \mathbf{a}^{3}$ and $\mathbf{2} \mathbf{b}^{4}$ were prepared by aminative cyclization of the parent dibromo derivatives following literature procedures. Aziridine 2c was synthesized in four steps from commercially available phenylglyoxylic acid (2d) as described below.

Chromatographic purification of compounds was carried out on silica gel $(60-200 \mu \mathrm{m})$. TLC analysis was performed on pre-loaded $(0.25 \mathrm{~mm})$ glass supported silica gel plates (Kieselgel 60$)$; compounds were visualized by exposure to UV light and by dipping the plates in $1 \%$ $\mathrm{Ce}\left(\mathrm{SO}_{4}\right) \cdot 4 \mathrm{H}_{2} \mathrm{O}, 2.5 \%\left(\mathrm{NH}_{4}\right)_{6} \mathrm{Mo}_{7} \mathrm{O}_{24} \cdot 4 \mathrm{H}_{2} \mathrm{O}$ in $10 \%$ sulphuric acid followed by heating on a hot plate.

${ }^{1} \mathrm{H}$ and ${ }^{13} \mathrm{C}$ NMR spectra were recorded in $\mathrm{CDCl}_{3}$ solution on 200 or $400 \mathrm{MHz}$ instruments. Multiplicity is given as $\mathrm{s}=$ singlet, $\mathrm{d}=$ doublet, $\mathrm{t}=$ triplet $\mathrm{q}=$ quartet, $\mathrm{m}=$ multiplet and $\mathrm{br}=\mathrm{broad}$ signal, and chemical shifts $(\delta)$ are reported in ppm downfield from TMS as internal standard. All coupling constants $(J)$ are given in $\mathrm{Hz}$. In ${ }^{13} \mathrm{C}$ NMR spectra of imidazoles $\mathbf{4 a - k}$, hydrogen multiplicity information from DEPT experiments has also been included. FT-IR spectra were obtained as Nujol mulls. Mass spectra were recorded in the EI mode (70eV). Melting points were determined in open capillary tubes and are uncorrected.

\section{Experimental procedures and spectral data.}

trans-3-Phenyl-2-aziridinecarboxylic acid methyl ester (2a) ${ }^{3}$

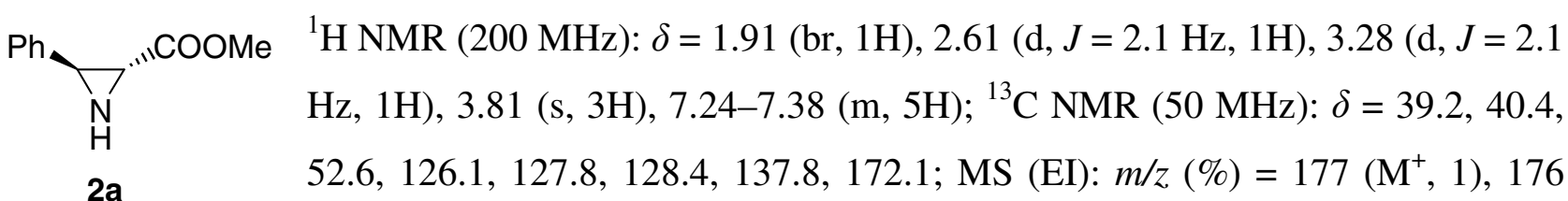
(1), 162 (12), 146 (15), 131 (7), 117 (100), 103 (7), 91 (45), 77 (12), 65 (10), 63 (11), 51 (11). Anal. Calcd. for $\mathrm{C}_{10} \mathrm{H}_{11} \mathrm{NO}_{2}$ : C, 67.78; H, 6.26; N, 7.90. Found: C, 67.55; H, 6.35; N, 7.79.

\section{2,2-Aziridinedicarboxylic acid dimethyl ester (2b) ${ }^{4}$}

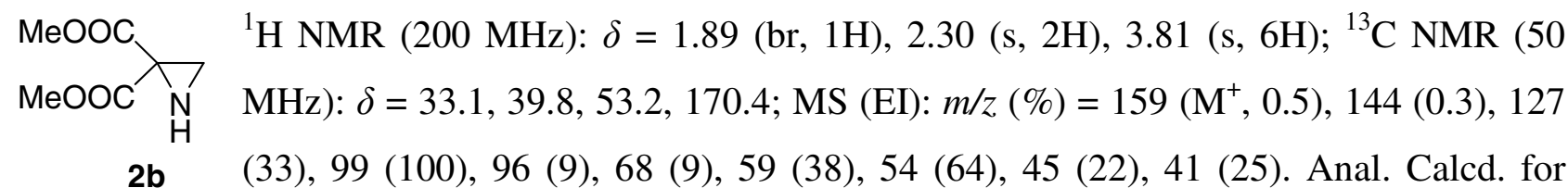
$\mathrm{C}_{6} \mathrm{H}_{9} \mathrm{NO}_{4}: \mathrm{C}, 45.28 ; \mathrm{H}, 5.70 ; \mathrm{N}, 8.80$. Found: C, 45.51; H, 5.58; N, 8.63. 


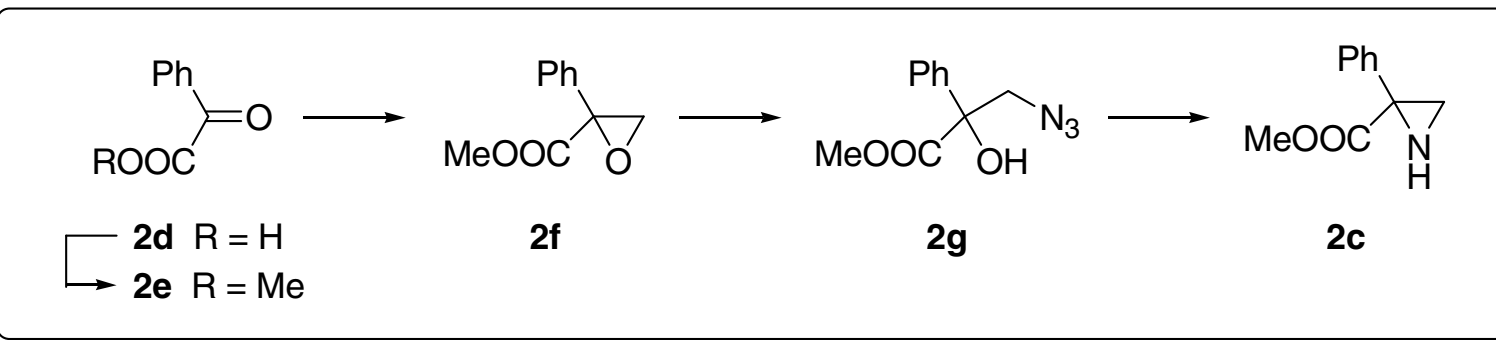

\section{2-Phenyl-2-oxiranecarboxylic acid methyl ester $(2 \mathrm{f})^{5}$}

$\begin{aligned} \text { MeOOC }_{\mathrm{O}}^{\mathrm{Ph}} & \begin{array}{l}\text { Methyl phenylglyoxylate } \\ \text { prepared solution of diazomethane }\end{array}{ }^{7}(43 \mathrm{mmol}) \text { in diethyl ether }(125 \mathrm{~mL}) \text { cooled to - } \\ \text { 2f } & 10{ }^{\circ} \mathrm{C} \text {, and the reaction mixture was warmed to } \mathrm{rt} \text { overnight until effervescence }\end{aligned}$ ceased. Concentration under reduced pressure gave a lemon yellow liquid which was purified by silica gel chromatography (light petroleum/diethyl ether 8:2) to afford the title epoxide as a pale yellow clear liquid (2.817 g, 73\% yield). ${ }^{1} \mathrm{H}$ NMR (200 MHz): $\delta=2.98(\mathrm{~d}, J=6.4 \mathrm{~Hz}, 1 \mathrm{H}), 3.44(\mathrm{~d}$, $J=6.4 \mathrm{~Hz}, 1 \mathrm{H}), 3.80(\mathrm{~s}, 3 \mathrm{H}), 7.20-7.60(\mathrm{~m}, 5 \mathrm{H}) ;{ }^{13} \mathrm{C} \mathrm{NMR}(50 \mathrm{MHz}): \delta=51.0,54.1,58.0,127.2$, 128.2, 128.6, 134.5, $\mathrm{C}=\mathrm{O}$ not seen; MS (EI): $m / z(\%)=178\left(\mathrm{M}^{+}, 10\right), 162$ (16), 146 (2), 131 (5), 119 (11), 105 (100), 103 (46), 91 (47), 77 (46), 63 (13), 51 (21), 45 (4). Anal. Calcd. for $\mathrm{C}_{10} \mathrm{H}_{10} \mathrm{O}_{3}$ : C, 67.41; H, 5.66. Found: C, 67.64; H, 5.52.

\section{3-Azido-2-hydroxy-2-phenylpropionic acid methyl ester (2g)}

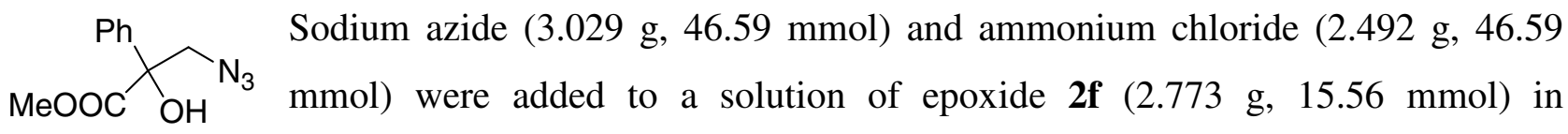
2g anhydrous $\mathrm{MeOH}(90 \mathrm{~mL})$, and the mixture was refluxed overnight. After pouring in distd. water $(400 \mathrm{~mL})$, the two layers were separated and the aqueous phase was extracted with ethyl acetate $(2 \times 125 \mathrm{~mL})$. The organic phases were pooled, dried over $\mathrm{Na}_{2} \mathrm{SO}_{4}$, filtered and concentrated in vacuo to yield azido alcohol $\mathbf{2 g}$ as an orange-brown liquid (3.415 $\mathrm{g}$, $99 \%$ yield) which was used for the next step without further purification. ${ }^{1} \mathrm{H}$ NMR $(200 \mathrm{MHz}): \delta=$ $3.62(\mathrm{~d}, J=12.6 \mathrm{~Hz}, 1 \mathrm{H}), 3.85(\mathrm{~s}, 3 \mathrm{H}), 3.86(\mathrm{~d}, J=12.6 \mathrm{~Hz}, 1 \mathrm{H}), 7.15-7.65(\mathrm{~m}, 5 \mathrm{H}) ;{ }^{13} \mathrm{C} \mathrm{NMR}(50$ $\mathrm{MHz}): \delta=53.6,58.5,79.4,125.3,128.6,130.0,138.3,173.3 ; \mathrm{MS}(\mathrm{EI}): m / z(\%)=165\left([\mathrm{M}-56]^{+}, 4\right)$, 162 (0.8), 136 (1), 105 (100), 91 (1), 77 (54), 65 (0.5), 63 (0.8), 59 (2), 51 (21).

\section{2-Phenyl-2-aziridinecarboxylic acid methyl ester (2c)}

MeOOC ${ }_{\text {2c }}^{\text {By analogy to the synthesis of 2-phenyl-2-aziridinecarboxylic acid ethyl ester, }}{ }^{8}$ azido
alcohol $2 \mathrm{~g}(3.415 \mathrm{~g}, 15.44 \mathrm{mmol})$ and triphenylphosphine $(4.06 \mathrm{~g}, 15.48 \mathrm{mmol})$ were 
effervescence ceased. The reaction mixture was then heated at reflux overnight, concentrated to dryness under reduced pressure, and the crude residue was purified by silica gel chromatography (using light petroleum/diethyl ether from 6:4 to $4: 6$ as the eluant) to afford the desired aziridine 2c $(1.479 \mathrm{~g}, 54 \%$ yield $)$ as a pale orange liquid. ${ }^{1} \mathrm{H}$ NMR $(200 \mathrm{MHz}): \delta=1.93(\mathrm{br}, 1 \mathrm{H}), 2.03(\mathrm{~d}, J=$ $1.4 \mathrm{~Hz}, 1 \mathrm{H}), 2.53(\mathrm{~d}, J=1.4 \mathrm{~Hz}, 1 \mathrm{H}), 3.73(\mathrm{~s}, 3 \mathrm{H}), 7.30-7.50(\mathrm{~m}, 5 \mathrm{H}) ;{ }^{13} \mathrm{C} \mathrm{NMR}(50 \mathrm{MHz}): \delta=$ 35.2, 41.3, 53.2, 127.8, 128.0, 129.2, 136.4, 173.6; MS (EI): $m / z(\%)=176\left([\mathrm{M}-1]^{+}, 6\right), 162(16)$, 145 (5), 117 (100), 104 (29), 91 (17), 77 (19), 65 (4), 63 (5), 51 (7). Anal. Calcd. for $\mathrm{C}_{10} \mathrm{H}_{11} \mathrm{NO}_{2}$ : C, 67.78; H, 6.26; N, 7.90. Found: C, 67.95; H, 6.45; N, 7.81.

\section{General procedure for the synthesis of a-aziridinohydrazones $3 a-k$.}

A mixture of 1,2-diaza-1,3-butadiene 1a-e $(1.1 \mathrm{mmol})$ and aziridine 2a-c $(1.0 \mathrm{mmol})$ was stirred under solvent-free conditions at $65^{\circ} \mathrm{C}$. After disappearance of the aziridine (monitored by silica gel TLC; for reaction times, see Table 1) the crude mixture was purified by column chromatography on silica gel to afford adducts 3a-k.

\section{trans-1-\{1-Methoxycarbonyl-2-[2-(methoxycarbonyl)hydrazono]propyl\}-3-phenyl-2- aziridinecarboxylic acid methyl ester (3a)}

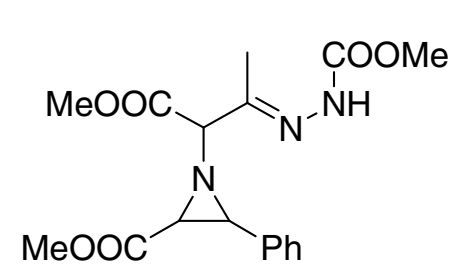

3a

a-Aziridinohydrazone 3a was isolated by column chromatography (cyclohexane/ethyl acetate 30:70) in 97\% yield as a mixture of inseparable isomers in 35:65 ratio (determined by ${ }^{1} \mathrm{H}$ NMR). White solid; ${ }^{1} \mathrm{H}$ NMR (400 MHz): $\delta=1.90$ and 1.99 (s, 3H), 2.73 and 3.30 (s, $1 \mathrm{H}), 2.98$ and $3.61(\mathrm{~d}, J=2.4 \mathrm{~Hz}, 1 \mathrm{H}), 3.66-3.82(\mathrm{~m}, 9 \mathrm{H}), 4.44$ and $4.74(\mathrm{~s}, 1 \mathrm{H}), 7.23-7.35(\mathrm{~m}, 5 \mathrm{H}), 7.70$ and $7.74(\mathrm{~s}, 1 \mathrm{H}) ;{ }^{13} \mathrm{C} \mathrm{NMR}(100 \mathrm{MHz}): \delta=12.0,42.6$ and 45.3, 46.5 and 47.8, 52.4, 52.6, 53.0, 68.3 and 68.5, 126.1, 126.6 and 127.8, 128.4 and 128.5, 137.3, 147.8 and 148.4, 154.1, 169.2 and 169.6. IR: $v=3295,1750,1727,1717 \mathrm{~cm}^{-1}$; MS (EI): $\mathrm{m} / z(\%)$ $363\left(\mathrm{M}^{+}, 6\right), 289$ (80), 257 (25), 176 (48), 116 (100). Anal.Calcd for $\mathrm{C}_{17} \mathrm{H}_{21} \mathrm{~N}_{3} \mathrm{O}_{6}$ : C, 56.19; H, 5.83; N, 11.56. Found: C, 56.03; H, 5.89; N, 11.41.

\section{1-\{1-Methoxycarbonyl-2-[2-(methoxycarbonyl)hydrazono]propyl\}-aziridine-2,2-dicarboxylic acid dimethyl ester (3b)}

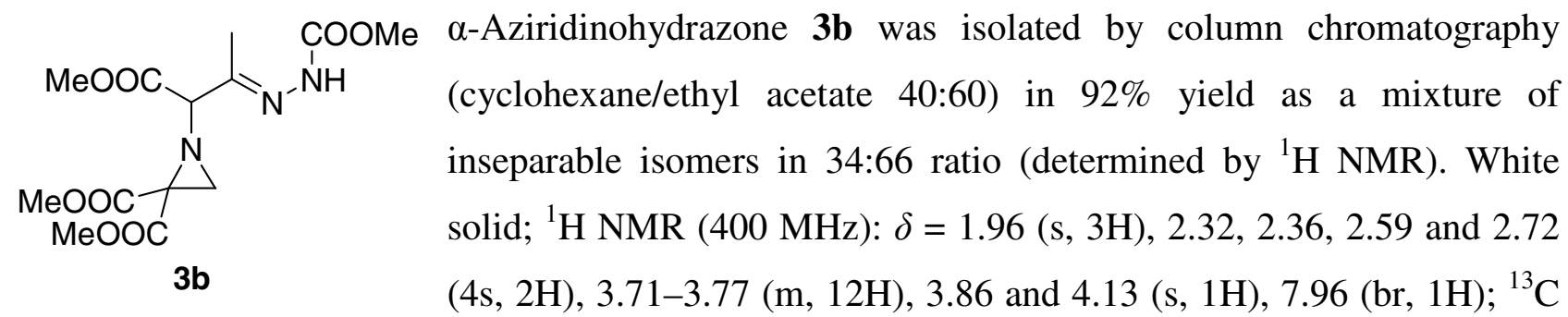


NMR (100 MHz): $\delta=11.7,37.6$ and 39.4, 44.9, 52.5, 52.9, 53.3, 69.7, 147.7, 154.1, 165.3, 166.9, 168.9. IR: $v=3305,1753,1721 \mathrm{~cm}^{-1}$; MS (EI): $m / z(\%) 345\left(\mathrm{M}^{+}, 7\right), 286$ (48), 254 (39), 198 (44), 142 (66), 113 (100). Anal.Calcd for $\mathrm{C}_{13} \mathrm{H}_{19} \mathrm{~N}_{3} \mathrm{O}_{8}$ : C, 45.22; H, 5.55; N, 12.17. Found: C, 45.43; H, $5.71 ; \mathrm{N}, 12.02$.

\section{trans-1-\{1-Ethoxycarbonyl-2-[2-(ethoxycarbonyl)hydrazono]propyl\}-3-phenyl-2-} aziridinecarboxylic acid methyl ester (3c)

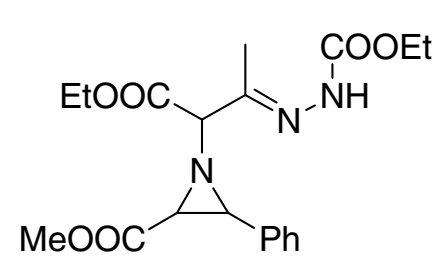

$3 c$

$\alpha$-Aziridinohydrazone 3c was isolated by column chromatography (cyclohexane/ethyl acetate 70:30) in $85 \%$ yield as a mixture of isomers in 25:75 ratio (determined by ${ }^{1} \mathrm{H}$ NMR) which were amenable to separation by flash chromatography. Minor isomer: white solid, mp: 78$82{ }^{\circ} \mathrm{C} ;{ }^{1} \mathrm{H}$ NMR $(400 \mathrm{MHz}): \delta=1.10(\mathrm{t}, J=7.2 \mathrm{~Hz}, 3 \mathrm{H}), 1.31(\mathrm{t}, J=7.2$ $\mathrm{Hz}, 3 \mathrm{H}), 1.99$ (s, 3H), 2.72 (s, 1H), 3.62 (d, $J=2.4 \mathrm{~Hz}, 1 \mathrm{H}), 3.80$ (s, 3H), 4.12 (q, J = $7.2 \mathrm{~Hz}, 2 \mathrm{H})$, $4.26(\mathrm{q}, J=7.2 \mathrm{~Hz}, 2 \mathrm{H}), 4.42(\mathrm{~s}, 1 \mathrm{H}), 7.21-7.34(\mathrm{~m}, 5 \mathrm{H}), 7.63(\mathrm{~s}, 1 \mathrm{H}) ;{ }^{13} \mathrm{C} \mathrm{NMR}(100 \mathrm{MHz}): \delta=$ 12.0, 14.0, 14.5, 42.9, 47.9, 52.6, 61.4, 61.9, 68.7, 126.7, 127.8, 128.3, 137.5, 147.8, 153.5, 168.4, 169.2; major isomer: white solid, mp: $102-106{ }^{\circ} \mathrm{C}$; ${ }^{1} \mathrm{H}$ NMR $(400 \mathrm{MHz}): \delta=1.20-1.32(\mathrm{~m}, 6 \mathrm{H})$ $1.90(\mathrm{~s}, 3 \mathrm{H}), 2.97(\mathrm{~d}, J=2.8 \mathrm{~Hz}, 1 \mathrm{H}), 3.31(\mathrm{~s}, 1 \mathrm{H}), 3.75(\mathrm{~s}, 3 \mathrm{H}), 4.14-4.27(\mathrm{~m}, 4 \mathrm{H}), 4.72(\mathrm{~s}, 1 \mathrm{H})$, 7.23--7.28 (m, 5H), $7.60(\mathrm{~s}, 1 \mathrm{H}) ;{ }^{13} \mathrm{C}$ NMR (100 MHz): $\delta=12.0,14.0,14.5,45.4,46.5,52.3,61.4$, 62.0, 68.3, 126.1, 127.7, 128.4, 137.3, 148.3, 153.6, 169.2. IR: $v=3235,1745,1735,1713 \mathrm{~cm}^{-1}$; MS (EI): $m / z(\%) 391\left(\mathrm{M}^{+}, 8\right), 314$ (26), 303 (84), 257 (16), 176 (52), 116 (100). Anal.Calcd for $\mathrm{C}_{19} \mathrm{H}_{25} \mathrm{~N}_{3} \mathrm{O}_{6}$ : C, 58.30; H, 6.44; N, 10.74. Found: C, 58.13; H, 6.39; N, 10.89.

1-\{1-Ethoxycarbonyl-2-[2-(ethoxycarbonyl)hydrazono]propyl\}-aziridine-2,2-dicarboxylic acid dimethyl ester (3d)

$\begin{array}{ll}\text { (cyclohexane/ethyl acetate } 50: 50) \text { in } 76 \% \text { yield as a mixture of } \\ \text { inseparable isomers in } 35: 65 \text { ratio (determined by }{ }^{1} \mathrm{H} \text { NMR). White } \\ \text { 3d } & \text { solid; }{ }^{1} \mathrm{H} \text { NMR }(400 \mathrm{MHz}): \delta=1.16-1.28(\mathrm{~m}, 6 \mathrm{H}), 1.94(\mathrm{~s}, 3 \mathrm{H}), 2.33, \\ & 2.57 \text { and } 2.72(3 \mathrm{~s}, 2 \mathrm{H}), 3.71(\mathrm{~s}, 3 \mathrm{H}), 3.75(\mathrm{~s}, 3 \mathrm{H}), 3.82(\mathrm{~s}, 1 \mathrm{H}), 4.12-\end{array}$ $4.22(\mathrm{~m}, 4 \mathrm{H}), 7.90(\mathrm{br}, 1 \mathrm{H}) ;{ }^{13} \mathrm{C} \mathrm{NMR}(100 \mathrm{MHz}): \delta=11.7,13.9,14.4,37.7$ and 39.4, 44.9 and $45.7,52.8,53.3,61.6,61.8,69.6,147.5,153.5,165.2,167.0,168.4$. IR: $v=3294,1739 \mathrm{~cm}^{-1}$; MS (EI): $m / z(\%) 373\left(\mathrm{M}^{+}, 6\right), 300$ (49), 212 (43), 145 (55), 129 (42), 113 (100). Anal.Calcd for $\mathrm{C}_{15} \mathrm{H}_{23} \mathrm{~N}_{3} \mathrm{O}_{8}$ : C, 48.25; H, 6.21; N, 11.25. Found: C, 48.43; H, 6.36; N, 11.46. 


\section{1-\{1-Ethoxycarbonyl-2-[2-(ethoxycarbonyl)hydrazono]propyl\}-2-phenyl-2-aziridinecarboxylic} acid methyl ester (3e)

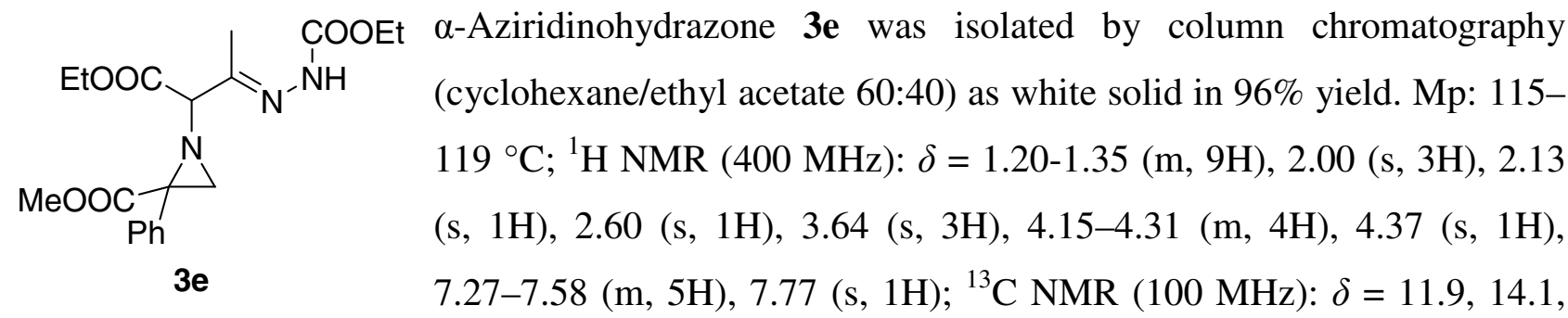
14.5, 40.4, 47.5, 52.7, 61.4, 62.0, 70.2, 127.6, 127.9, 128.8, 138.2, 148.4, 153.7, 169.5, 170.1. IR: $v$ = 3247, $1743 \mathrm{~cm}^{-1}$; MS (EI): m/z (\%) $391\left(\mathrm{M}^{+}, 1\right), 318$ (10), 303 (80), 242 (72), 196 (61), 176 (63), 103 (100). Anal.Calcd for $\mathrm{C}_{19} \mathrm{H}_{25} \mathrm{~N}_{3} \mathrm{O}_{6}$ : C, 58.30; H, 6.44; N, 10.74. Found: C, 58.17; H, 6.33; N, 10.26 .

trans-1-\{2-[2-(tert-Butoxycarbonyl)hydrazono]-1-(diethylcarbamoyl)propyl\}-3-phenyl-2aziridinecarboxylic acid methyl ester (3f)

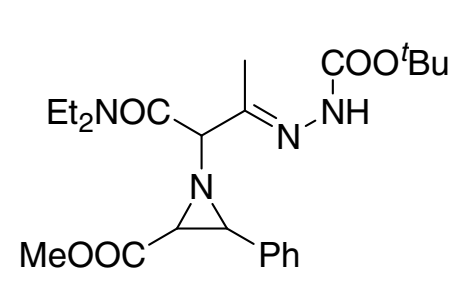

$3 f$

$\alpha$-Aziridinohydrazone $3 f$ was isolated by column chromatography (cyclohexane/ethyl acetate 65:35) in $81 \%$ yield as a mixture of isomers in 13:87 ratio (determined by ${ }^{1} \mathrm{H}$ NMR) which could be separated by flash chromatography. Minor isomer: white solid, mp: $112-116{ }^{\circ} \mathrm{C} ;{ }^{1} \mathrm{H}$ NMR (400 MHz): $\delta=1.04(\mathrm{t}, J=7.2 \mathrm{~Hz}, 3 \mathrm{H}), 1.12(\mathrm{t}, J=7.2 \mathrm{~Hz}, 3 \mathrm{H})$, 1.49 (s, 9H), 1.99 (s, 3H), 2.72 (s, 1H), 3.10-3.35 (m, 4H), 3.39 (s, 1H), 3.44-3.68 (m, 4H), 3.79 (s, $3 \mathrm{H}), 4.65(\mathrm{~s}, 1 \mathrm{H}), 7.24-7.45(\mathrm{~m}, 5 \mathrm{H}), 7.53(\mathrm{~s}, 1 \mathrm{H}) ;{ }^{13} \mathrm{C} \mathrm{NMR}(100 \mathrm{MHz}): \delta=11.4,12.8,14.2,28.2$, $40.5,41.5,43.4,47.2,52.4,66.2,81.0,126.9,127.5,128.3,137.4,148.6,152.2,167.2$, 169.0; major isomer: mp: $136-140{ }^{\circ} \mathrm{C} ;{ }^{1} \mathrm{H}$ NMR (400 MHz): $\delta=1.05-1.20(\mathrm{~m}, 6 \mathrm{H}) 1.48(\mathrm{~s}, 9 \mathrm{H}), 1.94(\mathrm{~s}, 3 \mathrm{H})$, 2.99 (s, 1H), 3.20-3.60 (m, 5H), 3.67 (s, 3H), $4.92(\mathrm{~s}, 1 \mathrm{H}), 7.20-7.30(\mathrm{~m}, 5 \mathrm{H}), 7.45(\mathrm{~s}, 1 \mathrm{H}) ;{ }^{13} \mathrm{C}$ NMR (100 MHz): $\delta=11.3,12.6,13.5,28.2,40.4,41.5,44.8,48.1,52.1,68.5,81.3,126.3,127.5$, 128.3, 138.1, 149.1, 152.3, 167.1, 169.8. IR: $v=3219,1728,1699,1658 \mathrm{~cm}^{-1}$; MS (EI): $m / z(\%)$ $446\left(\mathrm{M}^{+}, 2\right), 330$ (30), 290 (83), 176 (55), 116 (62), 100 (100). Anal.Calcd for $\mathrm{C}_{23} \mathrm{H}_{34} \mathrm{~N}_{4} \mathrm{O}_{5}$ : C, 61.86; H, 7.67; N, 12.55. Found: C, 61.65; H, 7.74; N, 12.76.

\section{1-\{2-[2-(tert-Butoxycarbonyl)hydrazono]-1-(diethylcarbamoyl)propyl\}-aziridine-2,2-} dicarboxylic acid dimethyl ester (3g)

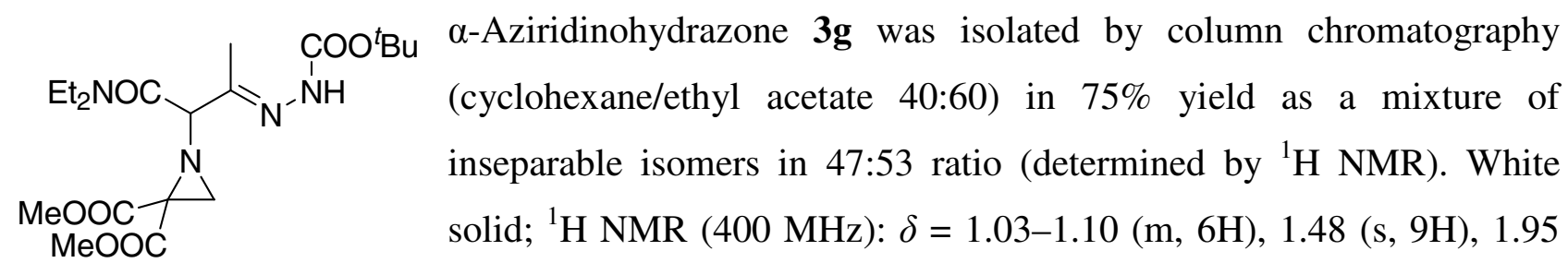


(s, 3H), 2.44 and $2.55(2 \mathrm{~s}, 2 \mathrm{H}), 2.55(\mathrm{br}, 1 \mathrm{H}), 3.19-3.52(\mathrm{~m}, 4 \mathrm{H}), 3.71(\mathrm{~s}, 3 \mathrm{H}), 3.80(\mathrm{~s}, 3 \mathrm{H}), 4.16$ (br, 1H), 7.56 (s, 1H); ${ }^{13} \mathrm{C}$ NMR (100 MHz): $\delta=11.1,12.8,14.0,28.2,38.8,40.5,41.4,45.6,52.7$, 53.2, 67.4, 81.2, 148.2, 152.2, 166.5, 167.2. IR: $v=3292,1760,1735,1659 \mathrm{~cm}^{-1}$; MS (EI): $\mathrm{m} / z(\%)$ $428\left(\mathrm{M}^{+}, 2\right), 272$ (45), 228 (49), 100 (100). Anal.Calcd for $\mathrm{C}_{19} \mathrm{H}_{32} \mathrm{~N}_{4} \mathrm{O}_{7}$ : C, 53.26; H, 7.53; N, 13.08. Found: C, 53.42; H, 7.36; N, 13.23.

\section{1-\{2-[2-(tert-Butoxycarbonyl)hydrazono]-1-(diethylcarbamoyl)propyl\}-2-phenyl-2-}

\section{aziridinecarboxylic acid methyl ester (3h)}

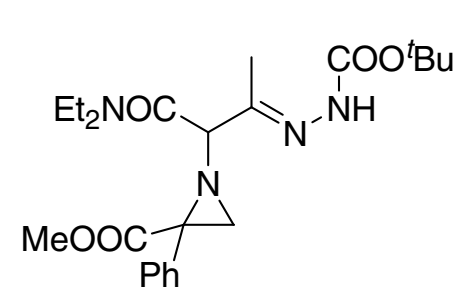

$3 h$

a-Aziridinohydrazone $\mathbf{3 h}$ was isolated by column chromatography (cyclohexane/ethyl acetate 60:40) as white solid in 78\% yield. Mp: 175-179 ${ }^{\circ} \mathrm{C} ;{ }^{1} \mathrm{H}$ NMR (400 MHz): $\delta=1.01-1.14(\mathrm{~m}, 6 \mathrm{H}), 1.52(\mathrm{~s}, 9 \mathrm{H})$, $2.00(\mathrm{~s}, 3 \mathrm{H}), 2.16(\mathrm{~s}, 1 \mathrm{H}), 2.69(\mathrm{~s}, 1 \mathrm{H}), 3.25-3.35(\mathrm{~m}, 4 \mathrm{H}), 3.57(\mathrm{~s}, 3 \mathrm{H})$, 4.57 (s, 1H), 7.33 (t, $J=7.2 \mathrm{~Hz}, 3 \mathrm{H}), 7.54(\mathrm{~s}, 1 \mathrm{H}), 7.77$ (d, $J=7.2 \mathrm{~Hz}$,

$2 \mathrm{H}) ;{ }^{13} \mathrm{C}$ NMR $(100 \mathrm{MHz}): \delta=11.3,12.7,13.5,28.2,40.3,41.6,46.9,52.6,69.8,81.3,127.3$, 127.8, 129.3, 138.7, 149.3, 152.4, 167.3, 170.9. IR: $v=3236,1726,1707,1656 \mathrm{~cm}^{-1}$; MS (EI): $\mathrm{m} / \mathrm{z}$ (\%) $446\left(\mathrm{M}^{+}, 1\right), 346$ (20), 230 (21), 290 (82), 246 (30), 214 (30), 100 (100). Anal.Calcd for $\mathrm{C}_{23} \mathrm{H}_{34} \mathrm{~N}_{4} \mathrm{O}_{5}$ : C, 61.86; H, 7.67; N, 12.55. Found: C, 61.63; H, 7.49; N, 12.77.

trans-1-\{1-Methoxycarbonyl-2-[2-(methoxycarbonyl)hydrazono]butyl\}-3-phenyl-2aziridinecarboxylic acid methyl ester (3i)

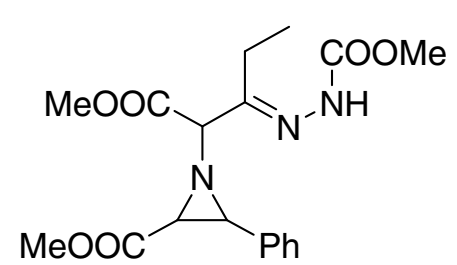

$3 \mathbf{i}$

a-Aziridinohydrazone 3i was isolated by column chromatography (cyclohexane/ethyl acetate 45:55) in $97 \%$ yield as a mixture of inseparable isomers in 41:59 ratio (determined by ${ }^{1} \mathrm{H}$ NMR). White solid; ${ }^{1} \mathrm{H}$ NMR (400 MHz): $\delta=0.93(\mathrm{t}, J=7.2 \mathrm{~Hz}, 3 \mathrm{H})$ and $1.20(\mathrm{t}, J=$ $7.2 \mathrm{~Hz}, 3 \mathrm{H}), 2.28$ and $2.44(\mathrm{~m}, 2 \mathrm{H}), 2.71$ and $3.58(\mathrm{~s}, 1 \mathrm{H}), 3.03$ and 3.29 $(\mathrm{t}, J=2.0 \mathrm{~Hz}, 1 \mathrm{H}), 3.66-3.83(\mathrm{~m}, 9 \mathrm{H}), 4.45$ and $4.74(\mathrm{~s}, 1 \mathrm{H}), 7.22-7.35(\mathrm{~m}, 5 \mathrm{H}), 7.78$ and $7.84(\mathrm{~s}$, $1 \mathrm{H}) ;{ }^{13} \mathrm{C}$ NMR (100 MHz): $\delta=9.4$ and 9.7, 19.6 and 20.0, 43.1 and 45.3, 47.0 and 48.0, 52.3, 52.6, 53.0, 68.6, 126.2, 126.5 and 127.8, 128.4, 137.3, 151.9 and 152.6, 154.1, 168.2 and 169.3, 169.8 and 170.0. IR: $v=3231,1745,1738,1712 \mathrm{~cm}^{-1}$; MS (EI): $\mathrm{m} / z(\%) 377\left(\mathrm{M}^{+}, 9\right), 303$ (100), $271(30)$, 176 (56), 156 (49), 116 (96). Anal.Calcd for $\mathrm{C}_{18} \mathrm{H}_{23} \mathrm{~N}_{3} \mathrm{O}_{6}$ : C, 57.29; H, 6.14; N, 11.13. Found: C, 57.38; H, 6.32; N, 11.01 . 


\section{1-\{2-[2-(Ethoxycarbonyl)hydrazono]-1-(methoxycarbonyl)butyl\}-aziridine-2,2-dicarboxylic} acid dimethyl ester (3j)

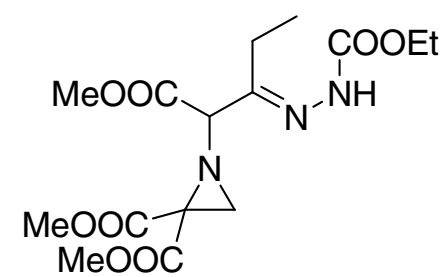

3j

a-Aziridinohydrazone $\mathbf{3 j}$ was isolated by column chromatography (cyclohexane/ethyl acetate 40:60) in $71 \%$ yield as a mixture of inseparable isomers in 50:50 ratio (determined by ${ }^{1} \mathrm{H}$ NMR). White solid; ${ }^{1} \mathrm{H}$ NMR (400 MHz): $\delta=1.06-1.20$ (m, 3H) 1.29 (m, 3H), 2.25$2.69(\mathrm{~m}, 4 \mathrm{H}), 3.73-3.82(\mathrm{~m}, 9 \mathrm{H}), 4.03$ and $4.17(\mathrm{~s}, 1 \mathrm{H}), 4.23(\mathrm{~m}, 2 \mathrm{H})$,

7.82 (br, $1 \mathrm{H}) ;{ }^{13} \mathrm{C}$ NMR (100 MHz): $\delta=9.3,14,5,19.6,38.4,39.5,45.4,52.4,52.9,53.4,61.4$, 61.9, 70.1, 151.3, 154.3, 166.4, 167.4, 169.1. IR: $v=3240,1754,1735,1709 \mathrm{~cm}^{-1}$; MS (EI): $\mathrm{m} / z$ (\%) 373 (M+, 2), 344 (6), 286 (9), 253 (9), 231 (8), 212 (10), 170 (18), 143 (38), 115 (100). Anal.Calcd for $\mathrm{C}_{15} \mathrm{H}_{23} \mathrm{~N}_{3} \mathrm{O}_{8}$ : C, 48.25; H, 6.21; N, 11.25. Found: C, 48.11; H, 6.04; N, 11.44.

\section{1-\{2-[2-(Ethoxycarbonyl)hydrazono]-1-(methoxycarbonyl)butyl\}-2-phenyl-2-} aziridinecarboxylic acid methyl ester (3k)

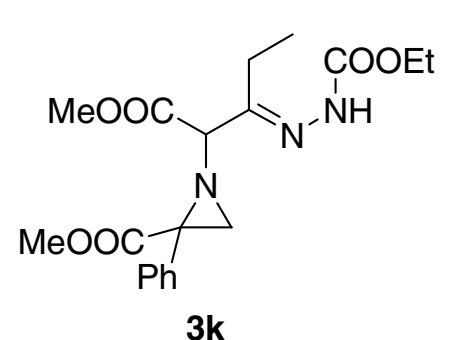

$\alpha$-Aziridinohydrazone $\mathbf{3 k}$ was isolated by column chromatography (cyclohexane/ethyl acetate 60:40) as white solid in 94\% yield. Mp: 110 $114{ }^{\circ} \mathrm{C} ;{ }^{1} \mathrm{H}$ NMR $(400 \mathrm{MHz}): \delta=1.08(\mathrm{t}, J=7.2 \mathrm{~Hz}, 3 \mathrm{H}) 1.33(\mathrm{t}, J=7.2$ $\mathrm{Hz}, 3 \mathrm{H}), 2.12$ (s, 1H), 2.35-2.60 (m, 2H), 2.60 (s, 1H), 3.63 (s, 3H), 3.71 $(\mathrm{s}, 3 \mathrm{H}), 4.29(\mathrm{q}, J=7.2 \mathrm{~Hz}, 2 \mathrm{H}), 4.34(\mathrm{~s}, 1 \mathrm{H}), 7.24-7.61(\mathrm{~m}, 5 \mathrm{H}), 7.90$ (s, 1H); ${ }^{13} \mathrm{C}$ NMR (100 MHz): $\delta=9.8,14.5,19.7,40.6,47.6,52.1,52.7,62.0,70.4,127.6,127.9$, 128.8, 131.8, 138.2, 152.5, 153.7, 170.1. IR: $v=3246,1749,1720,1703 \mathrm{~cm}^{-1}$; MS (EI): $\mathrm{m} / z(\%)$ $391\left(\mathrm{M}^{+}, 7\right), 332$ (12), 303 (100), 271 (28), 242 (74), 210 (61), 162 (97). Anal.Calcd for $\mathrm{C}_{19} \mathrm{H}_{25} \mathrm{~N}_{3} \mathrm{O}_{6}$ : C, 58.30; H, 6.44; N, 10.74. Found: C, 58.11; H, 6.67; N, 10.89.

\section{General procedure for the synthesis of imidazoles $4 a-k$.}

$\alpha$-Aziridinohydrazones 3a-k $(0.5 \mathrm{mmol})$ were dissolved in toluene and heated at reflux until TLC analysis showed disappearance of the starting material (for reaction times, see Table 1). The reaction mixture was then concentrated under reduced pressure and subjected to column chromatography on silica gel to yield imidazoles $\mathbf{4 a - k}$.

3-Methoxycarbonylmethyl-5-methyl-2-phenyl-3H-4-imidazolecarboxylic acid methyl ester (4a)<smiles>COC(=O)c1c(C)nc(-c2ccccc2)n1CC(C)=O</smiles>
Imidazole 4a was isolated by column chromatography (cyclohexane/ethyl acetate 55:45) as a white solid; mp: $85-89{ }^{\circ} \mathrm{C} ;{ }^{1} \mathrm{H}$ NMR $(400 \mathrm{MHz}): \delta=2.56$ (s, 3H), $3.82(\mathrm{~s}, 3 \mathrm{H}), 3.87(\mathrm{~s}, 3 \mathrm{H}), 4.92(\mathrm{~s}, 2 \mathrm{H}), 7.44-7.55(\mathrm{~m}, 5 \mathrm{H}) ;{ }^{13} \mathrm{C} \mathrm{NMR}$ 
(100 MHz): $\delta=15.7\left(\mathrm{CH}_{3}\right), 48.4\left(\mathrm{CH}_{2}\right), 51.4\left(\mathrm{CH}_{3}\right), 52.6\left(\mathrm{CH}_{3}\right), 119.6(\mathrm{C}), 128.9(\mathrm{CH}, 2 \mathrm{C}), 129.1$ (C), $129.3(\mathrm{CH}, 2 \mathrm{C}), 130.0(\mathrm{CH}), 147.7$ (C), 151.5 (C), 161.9 (C), 168.9 (C). IR: $v=1743,1692$ $\mathrm{cm}^{-1}$; MS (EI): m/z (\%) $288\left(\mathrm{M}^{+}, 100\right), 256$ (30), 229 (62), 198 (21), 169 (16). Anal.Calcd for $\mathrm{C}_{15} \mathrm{H}_{16} \mathrm{~N}_{2} \mathrm{O}_{4}$ : C, 62.49; H, 5.59; N, 9.72. Found: C, 62.35; H, 5.68; N, 9.57.

\section{2-[5-(Methoxycarbonyl)-4-methyl-1H-1-imidazolyl]malonic acid dimethyl ester (4b)}

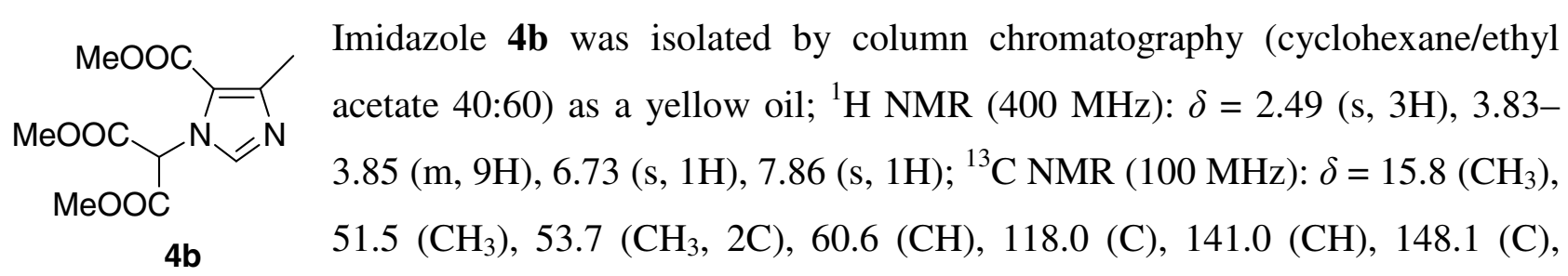
161.9 (C), 165.3 (C, 2C). IR: $v=1757,1703 \mathrm{~cm}^{-1}$; MS (EI): $m / z(\%) 270\left(\mathrm{M}^{+}, 43\right), 238(100), 211$ (35), 167 (25), 103 (25). Anal.Calcd for $\mathrm{C}_{11} \mathrm{H}_{14} \mathrm{~N}_{2} \mathrm{O}_{6}$ : C, 48.89; H, 5.22; N, 10.37. Found: C, 48.75; H, $5.11 ; \mathrm{N}, 10.59$.

\section{3-Methoxycarbonylmethyl-5-methyl-2-phenyl-3H-4-imidazolecarboxylic acid ethyl ester (4c)}

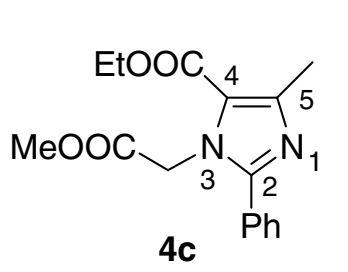

Imidazole $\mathbf{4 c}$ was isolated by column chromatography (cyclohexane/ethyl acetate 60:40) as a white solid; mp: $122-126{ }^{\circ} \mathrm{C} ;{ }^{1} \mathrm{H}$ NMR (400 MHz): $\delta=$ $1.36(\mathrm{t}, J=7.2 \mathrm{~Hz}, 3 \mathrm{H}), 2.56(\mathrm{~s}, 3 \mathrm{H}), 3.81(\mathrm{~s}, 3 \mathrm{H}), 4.31(\mathrm{q}, J=7.2 \mathrm{~Hz}, 2 \mathrm{H})$, $4.91(\mathrm{~s}, 2 \mathrm{H}), 7.42-7.55(\mathrm{~m}, 5 \mathrm{H}) ;{ }^{13} \mathrm{C} \mathrm{NMR}(100 \mathrm{MHz}): \delta=14.3\left(\mathrm{CH}_{3}\right), 15.8$ $\left(\mathrm{CH}_{3}\right), 48.4\left(\mathrm{CH}_{2}\right), 52.6\left(\mathrm{CH}_{3}\right), 60.4\left(\mathrm{CH}_{2}\right), 119.6(\mathrm{C}), 128.8(\mathrm{CH}, 2 \mathrm{C}), 129.2(\mathrm{CH}, 2 \mathrm{C}), 129.9(\mathrm{CH})$, 147.8 (C), 151.4 (C), 161.4 (C), 169.0 (C). The following HMBC correlations were diagnostic: $\mathrm{C}_{2}$ COOMe with C-2 and C-4, $\underline{\mathrm{C}}_{3}$ with C-4 and C-5. IR: $v=1759,1694 \mathrm{~cm}^{-1}$; MS (EI): $\mathrm{m} / z(\%)$ $302\left(\mathrm{M}^{+}, 100\right), 256$ (25), 229 (45), 215 (20), 198 (18), 171 (19). Anal.Calcd for $\mathrm{C}_{16} \mathrm{H}_{18} \mathrm{~N}_{2} \mathrm{O}_{4}$ : C, 63.56; H, 6.00; N, 9.27. Found: C, 63.75; H, 6.13; N, 9.39.

\section{2-[5-(Ethoxycarbonyl)-4-methyl-1H-1-imidazolyl]malonic acid dimethyl ester (4d)}

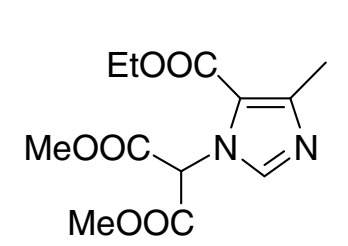

4d

Imidazole 4d was isolated by column chromatography (cyclohexane/ethyl acetate 50:50) as a yellow oil; ${ }^{1} \mathrm{H}$ NMR (400 MHz): $\delta=1.35(\mathrm{t}, J=7.2 \mathrm{~Hz}$, $3 \mathrm{H}), 2.47$ (s, 3H), 3.81 (s, 6H), 4.28 (q, J= $7.2 \mathrm{~Hz}, 2 \mathrm{H}), 6.72(\mathrm{~s}, 1 \mathrm{H}), 7.77$ (s,

$1 \mathrm{H}) ;{ }^{13} \mathrm{C}$ NMR $(100 \mathrm{MHz}): \delta=14.2\left(\mathrm{CH}_{3}\right), 15.8\left(\mathrm{CH}_{3}\right), 53.6\left(\mathrm{CH}_{3}, 2 \mathrm{C}\right), 60.5$ $(\mathrm{CH}), 60.6\left(\mathrm{CH}_{2}\right), 118.0(\mathrm{C}), 140.8(\mathrm{CH}), 147.8(\mathrm{C}), 161.4(\mathrm{C}), 165.3(\mathrm{C}, 2 \mathrm{C}) . \mathrm{IR}: v=1758,1701$, $1608 \mathrm{~cm}^{-1}$; MS (EI): m/z (\%) $284\left(\mathrm{M}^{+}, 55\right), 252$ (33), 238 (100), 224 (50), 211 (46), 149 (38), 137 (45). Anal.Calcd for $\mathrm{C}_{12} \mathrm{H}_{16} \mathrm{~N}_{2} \mathrm{O}_{6}$ : C, 50.70; H, 5.67; N, 9.85. Found: C, 50.89; H, 5.46; N, 9.69. 


\section{3-(Methoxycarbonyl-phenyl-methyl)-5-methyl-3H-4-imidazolecarboxylic acid ethyl ester (4e)}

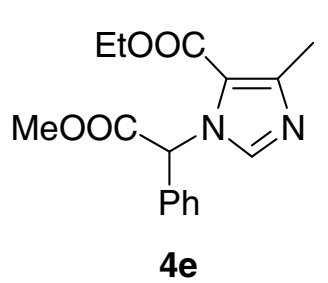

Imidazole 4e was isolated by column chromatography (cyclohexane/ethyl acetate 70:30) as a yellow oil; ${ }^{1} \mathrm{H}$ NMR (400 MHz): $\delta=1.36(\mathrm{t}, J=7.2 \mathrm{~Hz}$, 3H), 2.48 (s, 3H), $3.81(\mathrm{~s}, 3 \mathrm{H}), 4.31$ (q, $J=7.2 \mathrm{~Hz}, 2 \mathrm{H}), 6.61(\mathrm{~s}, 1 \mathrm{H}), 7.12$ (s,

$1 \mathrm{H}), 7.33-7.45(\mathrm{~m}, 5 \mathrm{H}) ;{ }^{13} \mathrm{C} \mathrm{NMR}(100 \mathrm{MHz}): \delta=14.2\left(\mathrm{CH}_{3}\right), 15.9\left(\mathrm{CH}_{3}\right)$, $52.8\left(\mathrm{CH}_{3}\right), 60.5\left(\mathrm{CH}_{2}\right), 63.2(\mathrm{CH}), 118.9(\mathrm{C}), 129.0(\mathrm{CH}, 2 \mathrm{C}), 129.4(\mathrm{CH}, 2 \mathrm{C}), 129.7(\mathrm{CH}), 132.9$ (C), $139.8(\mathrm{CH}), 148.4(\mathrm{C}), 161.6(\mathrm{C}), 169.8$ (C). IR: $v=1752,1702 \mathrm{~cm}^{-1}$; MS (EI): $\mathrm{m} / z(\%) 302$ (M+, 23), 270 (100), 256 (79), 242 (43), 135 (50), 121 (75), 105 (35). Anal.Calcd for $\mathrm{C}_{16} \mathrm{H}_{18} \mathrm{~N}_{2} \mathrm{O}_{4}$ : C, 63.56; H, 6.00; N, 9.27. Found: C, 63.39; H, 5.88; N, 9.43.

\section{2-[5-Diethylcarbamoyl-4-methyl-2-phenyl-1H-1-imidazolyl]acetic acid methyl ester (4f)}

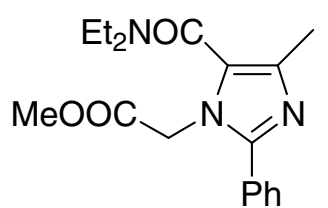

$4 \mathbf{f}$

Imidazole $\mathbf{4 f}$ was isolated by column chromatography (cyclohexane/ethyl acetate 35:65) as a white solid; mp: $99-103{ }^{\circ} \mathrm{C} ;{ }^{1} \mathrm{H}$ NMR (400 MHz): $\delta=1.18$ (t, $J=7.2 \mathrm{~Hz}, 6 \mathrm{H}), 2.26(\mathrm{~s}, 3 \mathrm{H}), 3.54(\mathrm{q}, J=7.2 \mathrm{~Hz}, 2 \mathrm{H}), 3.71(\mathrm{~s}, 3 \mathrm{H}), 4.86$ (s, 2H), 7.35-7.48 (m, 5H); ${ }^{13} \mathrm{C}$ NMR (100 MHz): $\delta=13.0\left(\mathrm{CH}_{3}, 2 \mathrm{C}\right), 13.9$ $\left(\mathrm{CH}_{3}\right), 38.3\left(\mathrm{CH}_{2}\right), 42.0\left(\mathrm{CH}_{2}\right), 46.4\left(\mathrm{CH}_{2}\right), 52.5\left(\mathrm{CH}_{3}\right), 123.7(\mathrm{C}), 128.7(\mathrm{CH}, 2 \mathrm{C}), 129.0(\mathrm{CH}, 2 \mathrm{C})$, $129.3(\mathrm{C}), 129.4(\mathrm{CH}), 136.6(\mathrm{C}), 149.1$ (C), 162.9 (C), 168.7 (C). IR: $v=1751,1609 \mathrm{~cm}^{-1}$; MS (EI): $m / z(\%) 329\left(\mathrm{M}^{+}, 49\right), 356$ (15), 230 (100), 171 (23). Anal.Calcd for $\mathrm{C}_{18} \mathrm{H}_{23} \mathrm{~N}_{3} \mathrm{O}_{3}: \mathrm{C}, 65.63 ; \mathrm{H}$, 7.04; N, 12.76. Found: C, 65.49; H, 6.98; N, 12.63.

\section{2-[5-Diethylcarbamoyl-4-methyl-1H-1-imidazolyl]malonic acid dimethyl ester (4g)}

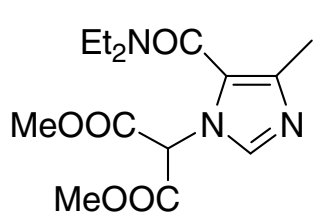

$4 \mathrm{~g}$

Imidazole $\mathbf{4 g}$ was isolated by column chromatography (cyclohexane/ethyl acetate15:85) as a yellow oil; ${ }^{1} \mathrm{H}$ NMR (400 MHz): $\delta=1.13(\mathrm{~s}, 6 \mathrm{H}), 2.20(\mathrm{~s}$, $3 \mathrm{H}), 3.47$ (s, 2H), $3.81(\mathrm{~s}, 6 \mathrm{H}), 5.81(\mathrm{~s}, 1 \mathrm{H}), 7.77(\mathrm{~s}, 1 \mathrm{H}) ;{ }^{13} \mathrm{C}$ NMR $(100$ $\mathrm{MHz}): \delta=13.6\left(\mathrm{CH}_{3}, 2 \mathrm{C}\right), 14.1\left(\mathrm{CH}_{3}\right), 39.4\left(\mathrm{CH}_{2}\right), 43.1\left(\mathrm{CH}_{2}\right), 53.6\left(\mathrm{CH}_{3}\right.$, 2C), $60.2(\mathrm{CH}), 122.7(\mathrm{C}), 137.0(\mathrm{C}), 138.5(\mathrm{CH}), 162.4(\mathrm{C}), 165.2(\mathrm{C}, 2 \mathrm{C}) . \mathrm{IR}: v=1756,1627 \mathrm{~cm}^{-}$

1; MS (EI): $m / z(\%) 311\left(\mathrm{M}^{+}, 53\right), 296$ (29), 279 (31), 239 (49), 212 (97), 149 (100). Anal.Calcd for $\mathrm{C}_{14} \mathrm{H}_{21} \mathrm{~N}_{3} \mathrm{O}_{5}$ : C, 54.01; H, 6.80; N, 13.50. Found: C, 54.09; H, 6.68; N, 13.72.

\section{2-[5-Diethylcarbamoyl-4-methyl-1H-1-imidazolyl]-2-phenylacetic acid methyl (4h)}

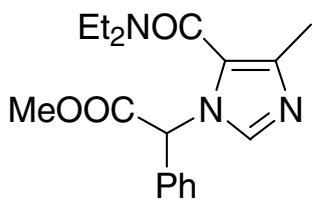

$4 \mathrm{~h}$ Imidazole 4h was isolated by column chromatography (ethyl acetate) as a yellow oil; ${ }^{1} \mathrm{H}$ NMR (400 MHz): $\delta=1.08$ (s, 6H), $2.16(\mathrm{~s}, 3 \mathrm{H}), 3.41(\mathrm{~s}, 4 \mathrm{H})$, $3.70(\mathrm{~s}, 3 \mathrm{H}), 6.17(\mathrm{~s}, 1 \mathrm{H}), 7.28-7.40(\mathrm{~m}, 6 \mathrm{H}) ;{ }^{13} \mathrm{C} \mathrm{NMR}(100 \mathrm{MHz}): \delta=13.1$ $\left(\mathrm{CH}_{3}, 2 \mathrm{C}\right), 13.9\left(\mathrm{CH}_{3}\right), 39.0\left(\mathrm{CH}_{2}\right), 42.5\left(\mathrm{CH}_{2}\right), 52.7\left(\mathrm{CH}_{3}\right), 61.8(\mathrm{CH}), 122.8$ (C), $128.5(\mathrm{CH}, 2 \mathrm{C}), 129.2(\mathrm{CH}), 129.4(\mathrm{CH}, 2 \mathrm{C}), 133.3(\mathrm{C}), 137.2(\mathrm{C}), 137.4(\mathrm{CH}), 162.9(\mathrm{C})$, 
169.4 (C). IR: $v=1751,1618 \mathrm{~cm}^{-1}$; MS (EI): $\mathrm{m} / z(\%) 329\left(\mathrm{M}^{+}, 42\right), 256$ (77), 240 (17), 198 (24), 171 (20), 121 (100). Anal.Calcd for $\mathrm{C}_{18} \mathrm{H}_{23} \mathrm{~N}_{3} \mathrm{O}_{3}$ : C, 65.63; H, 7.04; N, 12.76. Found: C, 65.49; H, $7.12 ; \mathrm{N}, 12.85$.

\section{5-Ethyl-3-methoxycarbonylmethyl-2-phenyl-3H-4-imidazolecarboxylic acid methyl ester (4i)}

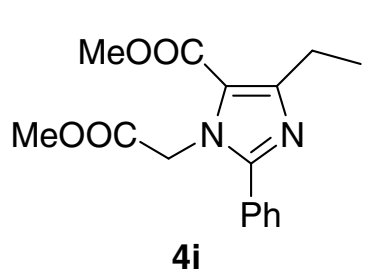

Imidazole 4i was isolated by column chromatography (cyclohexane/ethyl acetate 75:25) as a white solid; mp: $97-101{ }^{\circ} \mathrm{C} ;{ }^{1} \mathrm{H}$ NMR (400 MHz): $\delta=$ $1.27(\mathrm{t}, J=7.2 \mathrm{~Hz}, 3 \mathrm{H}), 2.93(\mathrm{q}, J=7.2 \mathrm{~Hz}, 2 \mathrm{H}), 3.78(\mathrm{~s}, 3 \mathrm{H}), 3.84(\mathrm{~s}, 3 \mathrm{H})$, 4.88 (s, 2H), 7.40-7.54 (m, 5H); ${ }^{13} \mathrm{C}$ NMR (100 MHz): $\delta=13.7\left(\mathrm{CH}_{3}\right), 22.8$

$\left(\mathrm{CH}_{2}\right), 48.4\left(\mathrm{CH}_{2}\right), 51.3\left(\mathrm{CH}_{3}\right), 52.5\left(\mathrm{CH}_{3}\right), 118.7(\mathrm{C}), 128.4(\mathrm{C}), 128.7(\mathrm{CH}, 2 \mathrm{C}), 129.2(\mathrm{CH}, 2 \mathrm{C})$, 129.8 (CH), 151.7 (C), 153.3 (C), 161.8 (C), 169.0 (C). IR: $v=1759,1708 \mathrm{~cm}^{-1}$; MS (EI): $m / z(\%)$ $302\left(\mathrm{M}^{+}\right.$, 54), 287 (7), 270 (13), 243 (100). Anal.Calcd for $\mathrm{C}_{16} \mathrm{H}_{18} \mathrm{~N}_{2} \mathrm{O}_{4}$ : C, 63.56; H, 6.00; N, 9.27. Found: C, 63.38; H, 5.87; N, 9.41.

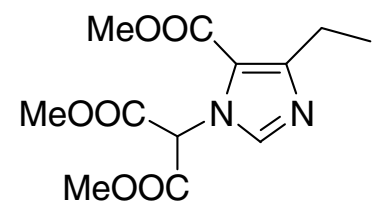

2-[4-Ethyl-5-(methoxycarbonyl)-1H-1-imidazolyl]malonic acid dimethyl ester (4j)

4j

Imidazole $\mathbf{4 j}$ was isolated by column chromatography (cyclohexane/ethyl acetate 60:40) as a yellow oil; ${ }^{1} \mathrm{H} \mathrm{NMR}(400 \mathrm{MHz}): \delta=1.25(\mathrm{t}, J=7.2 \mathrm{~Hz}$, $3 \mathrm{H}), 2.89$ (q, $J=7.2 \mathrm{~Hz}, 2 \mathrm{H}), 3.84(\mathrm{~s}, 6 \mathrm{H}), 3.85(\mathrm{~s}, 3 \mathrm{H}), 6.74(\mathrm{~s}, 1 \mathrm{H}), 7.81$ (s, 1H); ${ }^{13} \mathrm{C}$ NMR (100 MHz): $\delta=13.4\left(\mathrm{CH}_{3}\right), 22.8\left(\mathrm{CH}_{2}\right), 51.5\left(\mathrm{CH}_{3}\right), 53.6$ $\left(\mathrm{CH}_{3}, 2 \mathrm{C}\right), 60.6(\mathrm{CH}), 117.2(\mathrm{C}), 141.2(\mathrm{CH}), 153.5(\mathrm{C}), 161.9(\mathrm{C}), 165.3(\mathrm{C}, 2 \mathrm{C}) . \mathrm{IR}: v=1758$, $1708 \mathrm{~cm}^{-1}$; MS (EI): m/z (\%) $284\left(\mathrm{M}^{+}, 18\right), 252$ (45), 225 (100), 192 (15), 121 (30). Anal.Calcd for $\mathrm{C}_{12} \mathrm{H}_{16} \mathrm{~N}_{2} \mathrm{O}_{6}$ : C, 50.70; H, 5.67; N, 9.85. Found: C, 50.56; H, 5.84; N, 9.71.

\section{5-Ethyl-3-(methoxycarbonyl-phenyl-methyl)-3H-4-imidazolecarboxylic acid methyl ester (4k)}

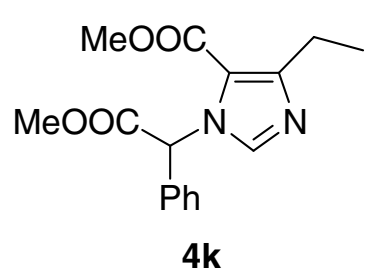

Imidazole 4k was isolated by column chromatography (cyclohexane/ethyl acetate 60:40) as a yellow oil; ${ }^{1} \mathrm{H}$ NMR (400 MHz): $\delta=1.23(\mathrm{t}, J=7.2 \mathrm{~Hz}$, $3 \mathrm{H}), 2.88(\mathrm{q}, J=7.2 \mathrm{~Hz}, 2 \mathrm{H}), 3.80(\mathrm{~s}, 3 \mathrm{H}), 3.87$ (s, 3H), 6.59 (s, 1H), 7.15 $(\mathrm{s}, 1 \mathrm{H}), 7.34-7.47(\mathrm{~m}, 5 \mathrm{H}) ;{ }^{13} \mathrm{C} \mathrm{NMR}(100 \mathrm{MHz}): \delta=13.5\left(\mathrm{CH}_{3}\right), 22.8$ $\left(\mathrm{CH}_{2}\right), 51.4\left(\mathrm{CH}_{3}\right), 52.9\left(\mathrm{CH}_{3}\right), 63.3(\mathrm{CH}), 118.0(\mathrm{C}), 129.1(\mathrm{CH}, 2 \mathrm{C}), 129.5(\mathrm{CH}, 2 \mathrm{C}), 129.8(\mathrm{CH})$, 132.7 (C), $140.1(\mathrm{CH}), 154.0(\mathrm{C}), 162.0(\mathrm{C}), 169.8$ (C). IR: $v=1761,1702 \mathrm{~cm}^{-1}$; MS (EI): $\mathrm{m} / z(\%)$ $302\left(\mathrm{M}^{+}, 7\right), 270$ (68), 255 (30), 210 (22), 149 (11), 121 (100). Anal.Calcd for $\mathrm{C}_{16} \mathrm{H}_{18} \mathrm{~N}_{2} \mathrm{O}_{4}$ : C, 63.56; H, 6.00; N, 9.27. Found: C, 63.74; H, 5.87; N, 9.11. 


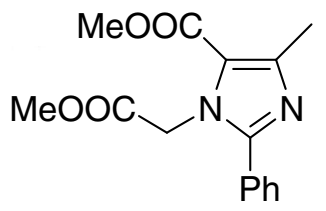

$4 a$
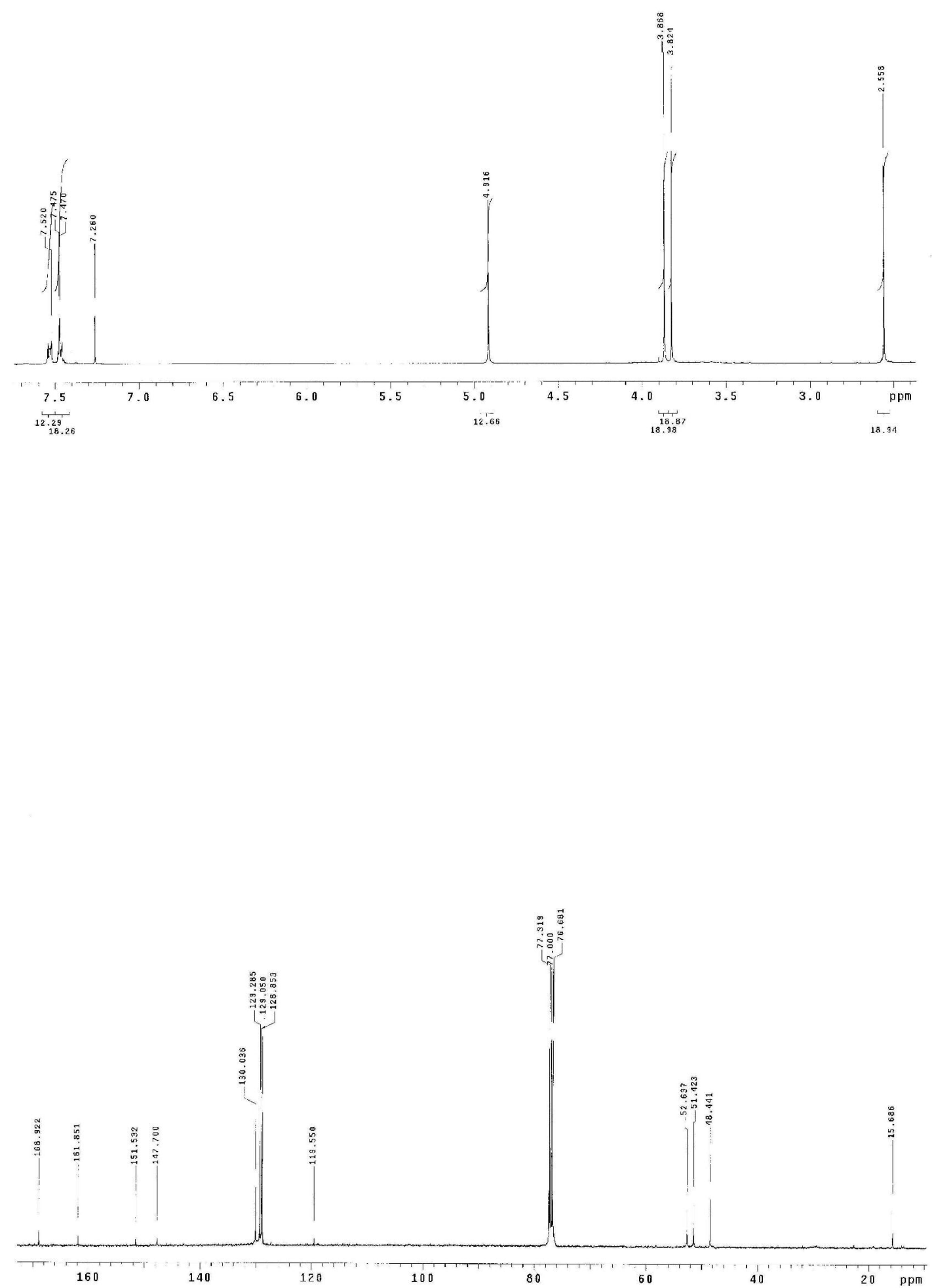


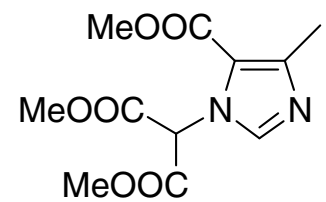

$4 b$
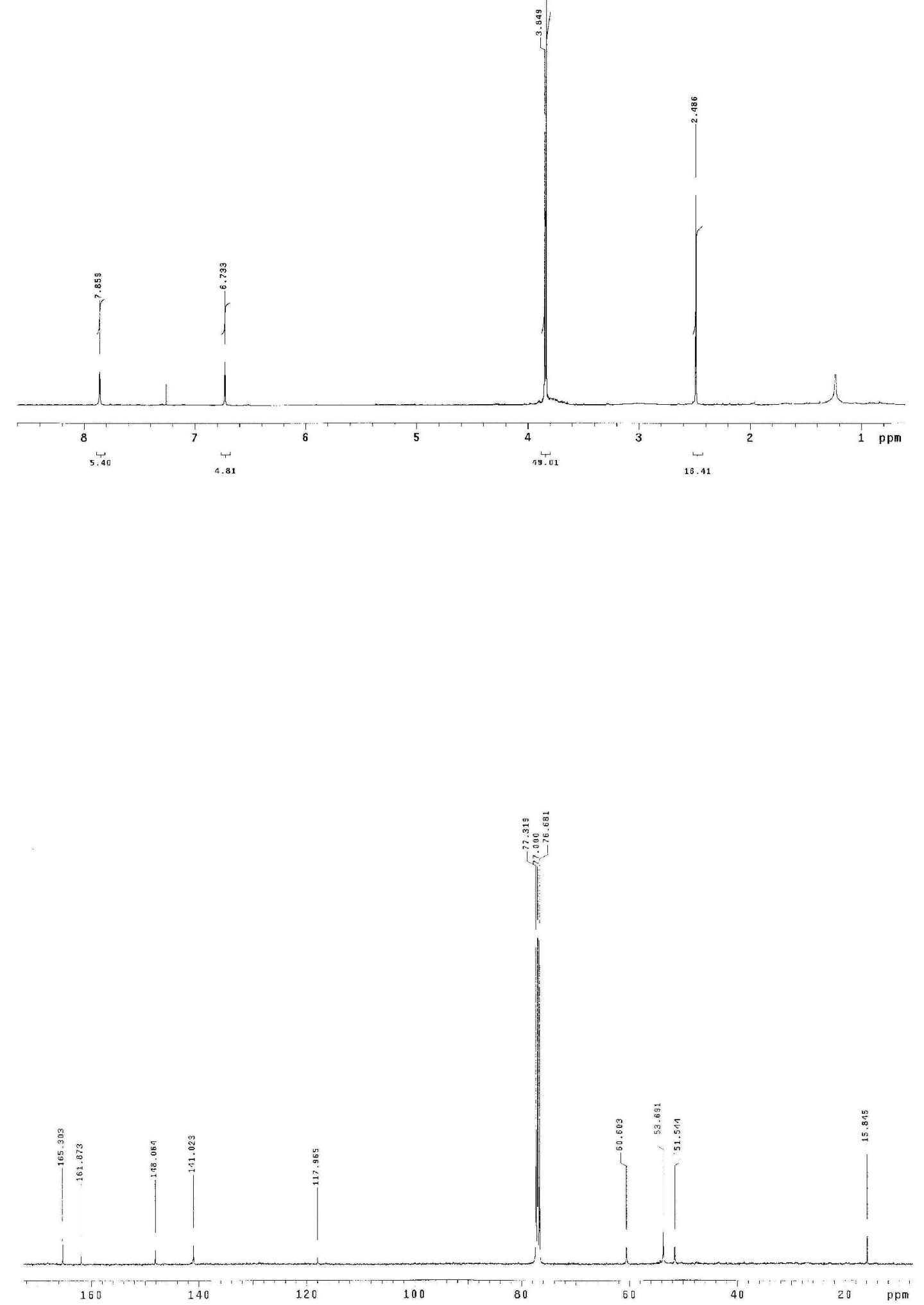
S14
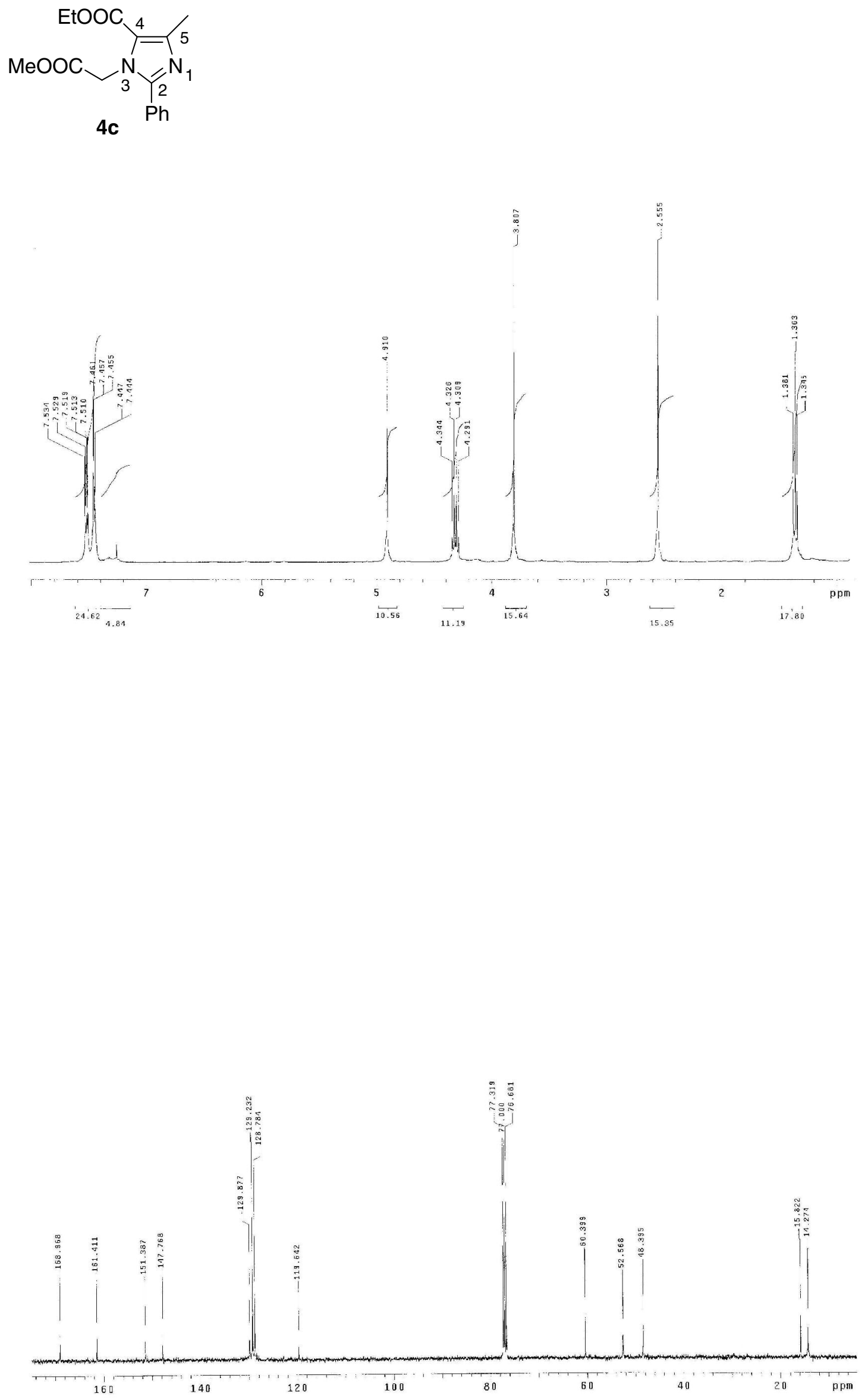
STANUARD IH OBSERYE

Pulse sequence: ghinqc_dō

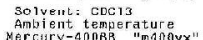

\section{Relax de $1 a y, 1.500500$}

Wid dit ime $0.234 \mathrm{sec}$

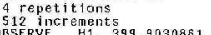

HAT
DATA PROCESSTWG
ST

Sa Sine bel1 0.137 sec
f1 DATA FROCESING

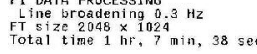

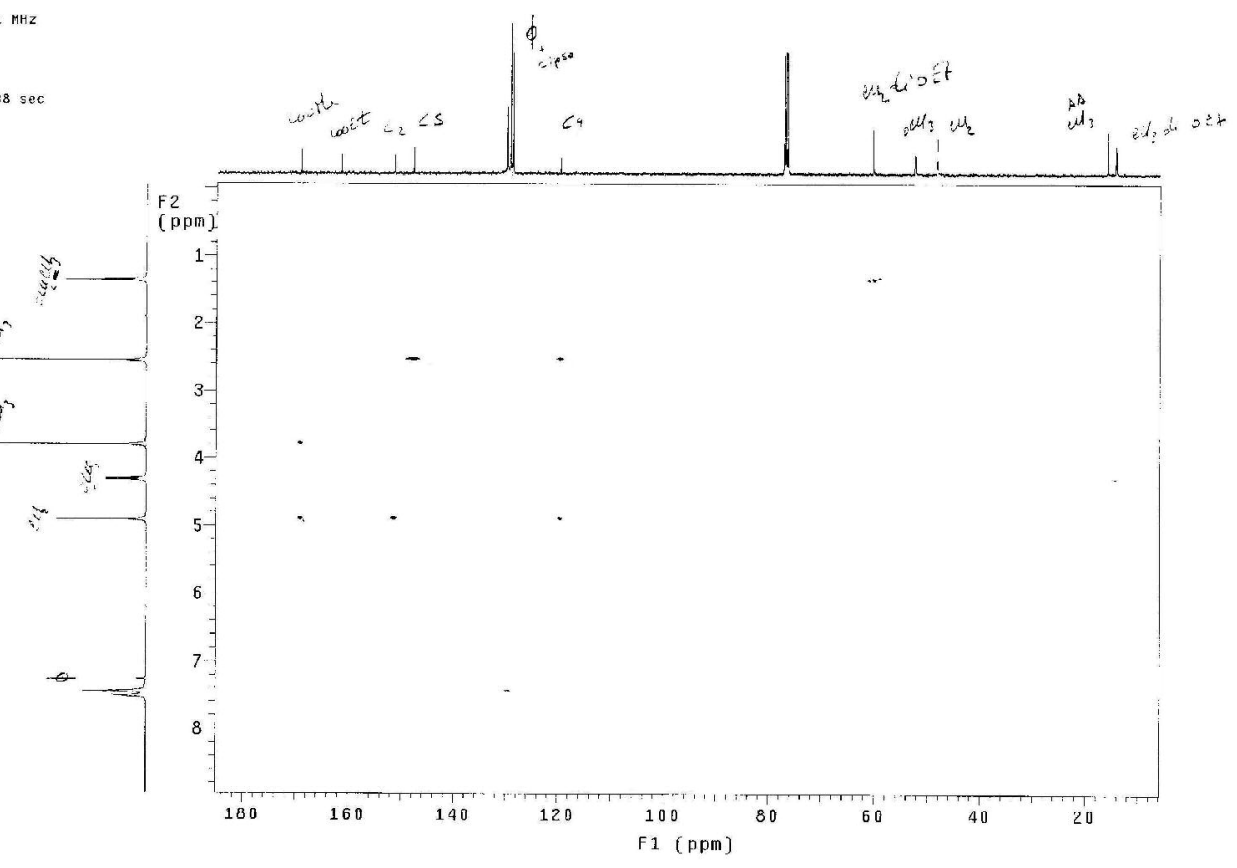


S16
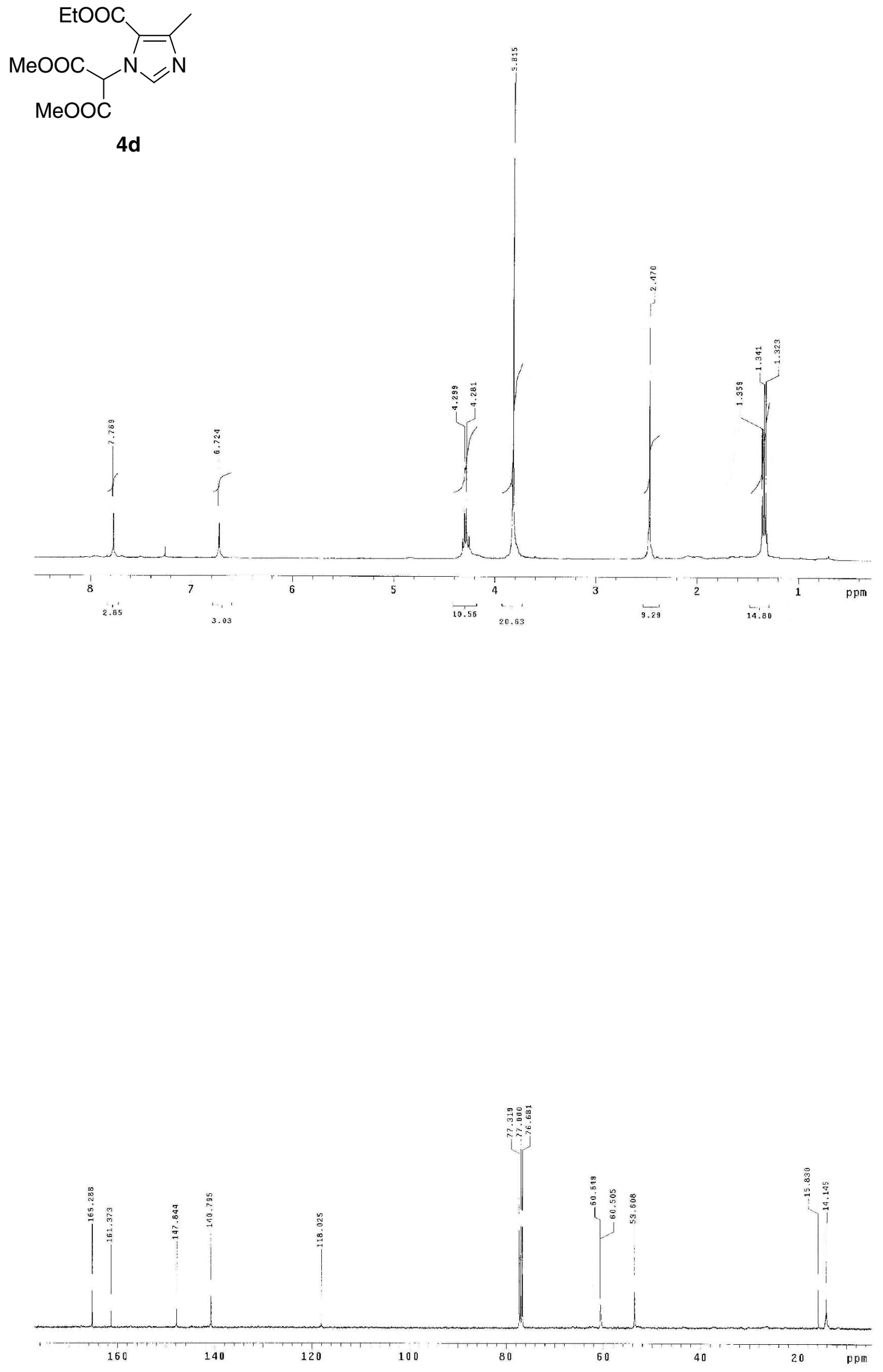


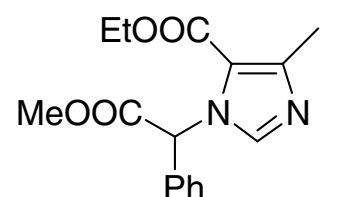

$\mathrm{Ph}$

$4 e$
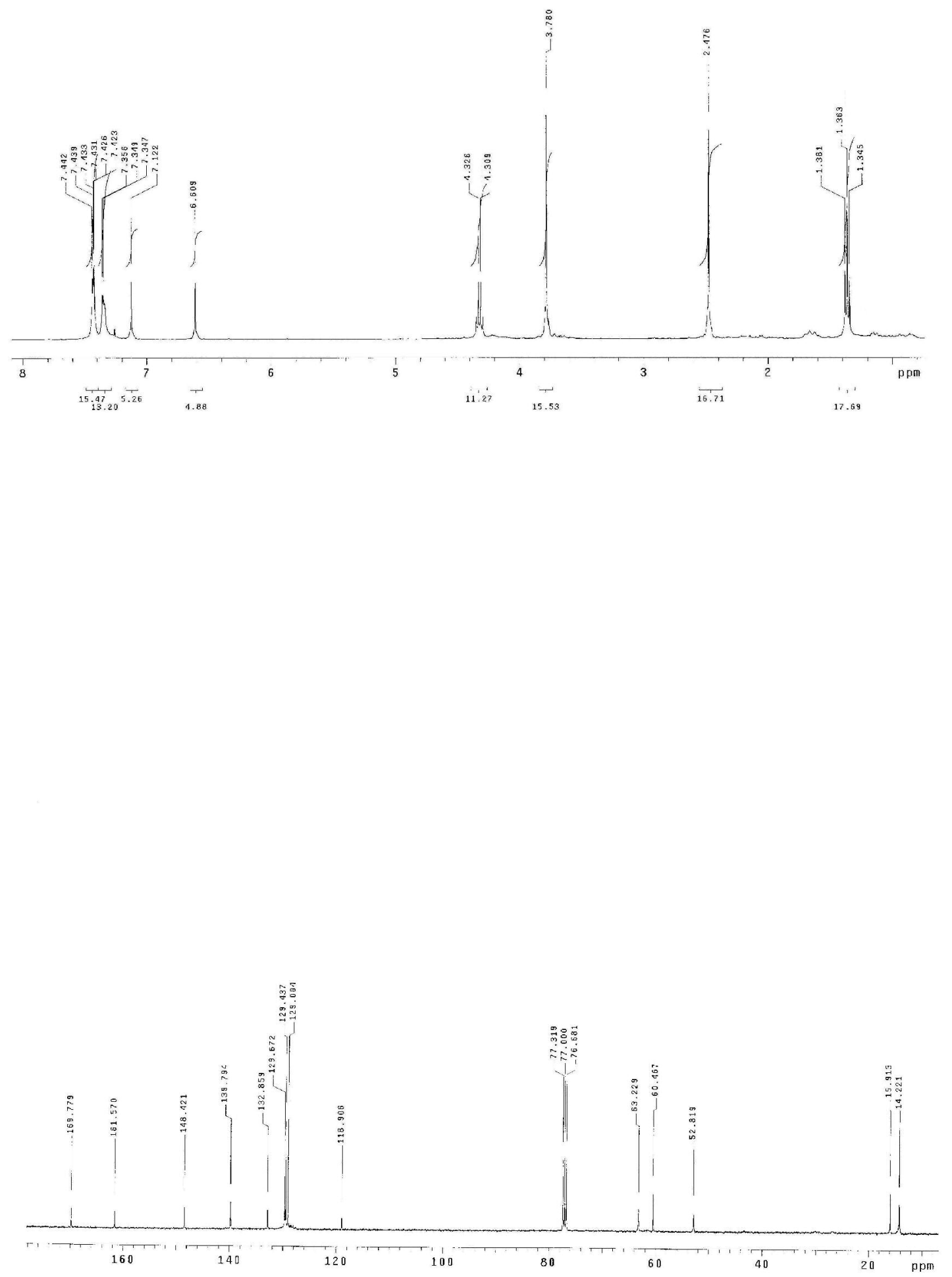

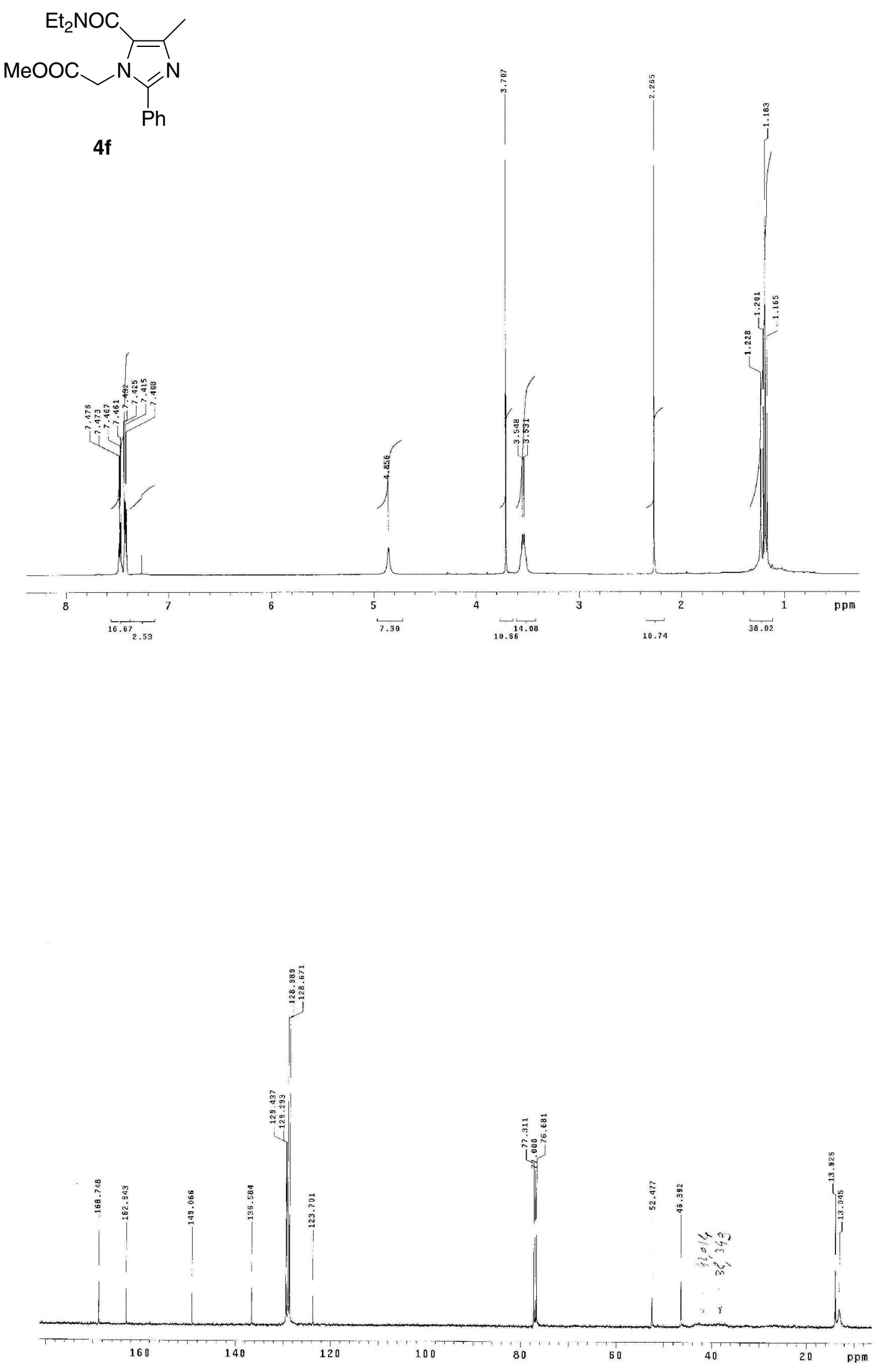

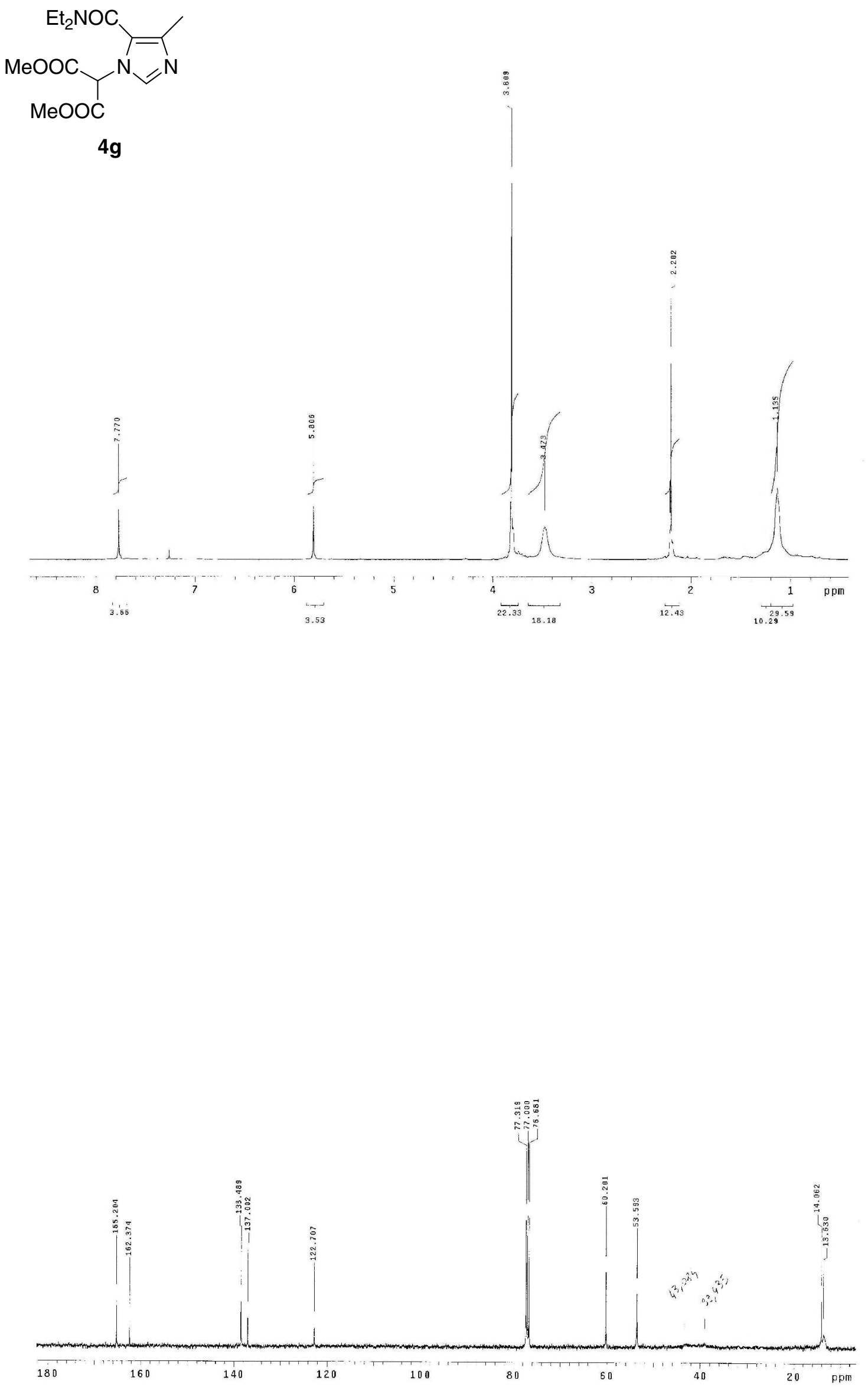


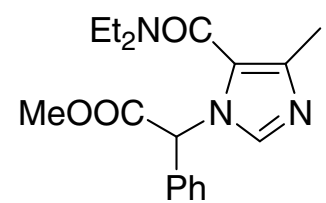

4h
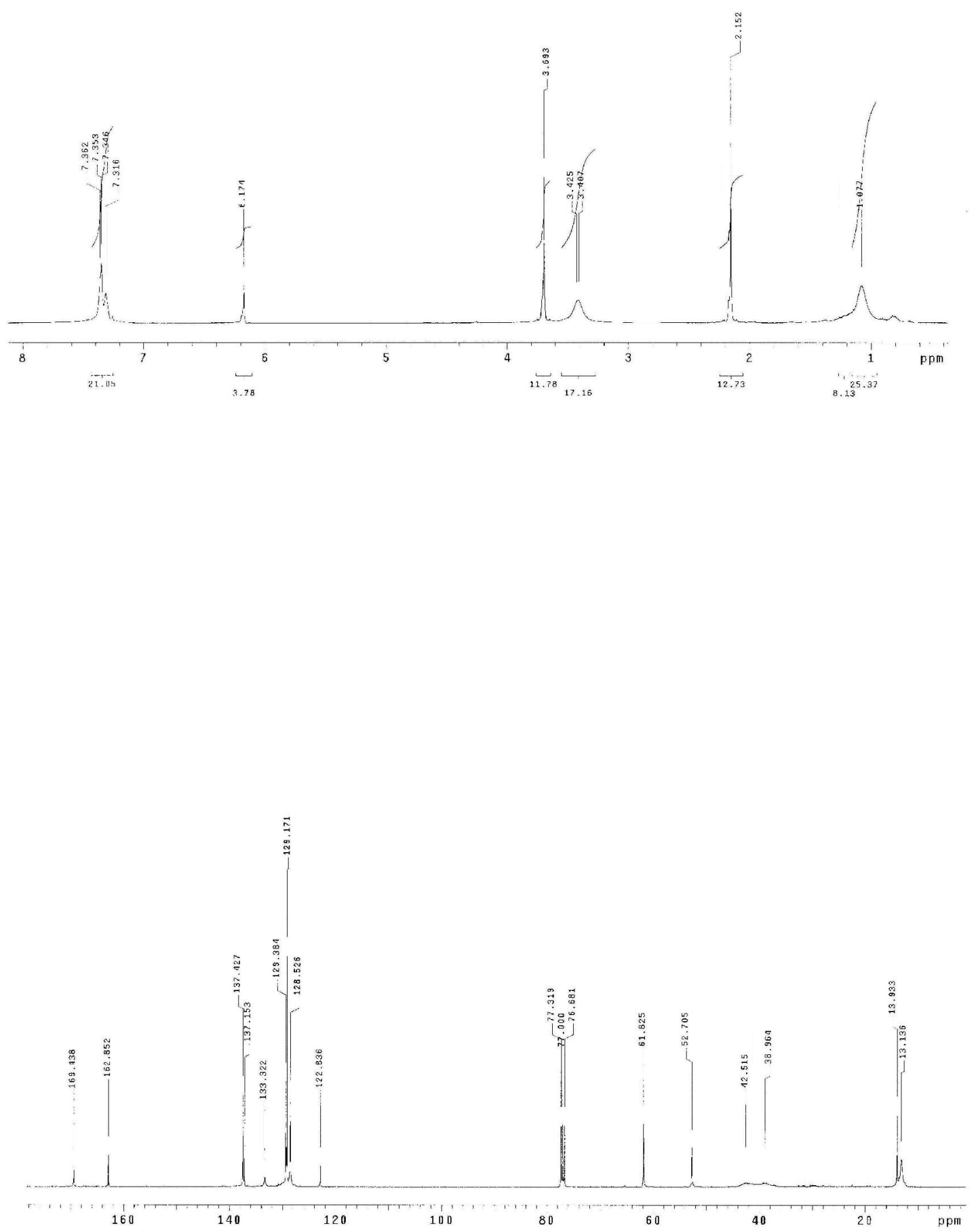


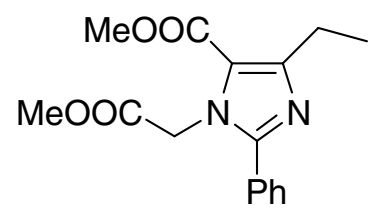

$4 i$
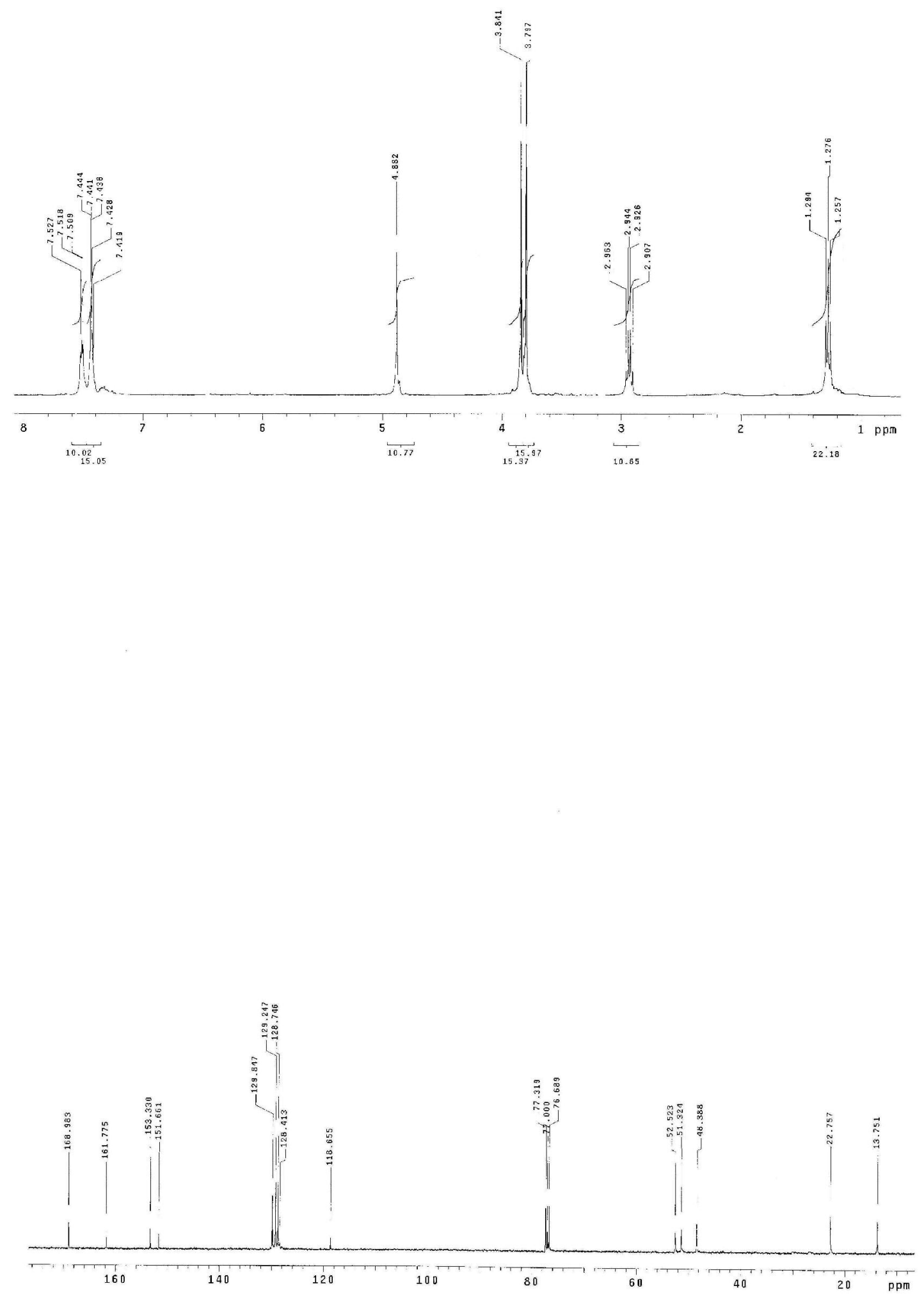
MeOOCLOCOC

4j
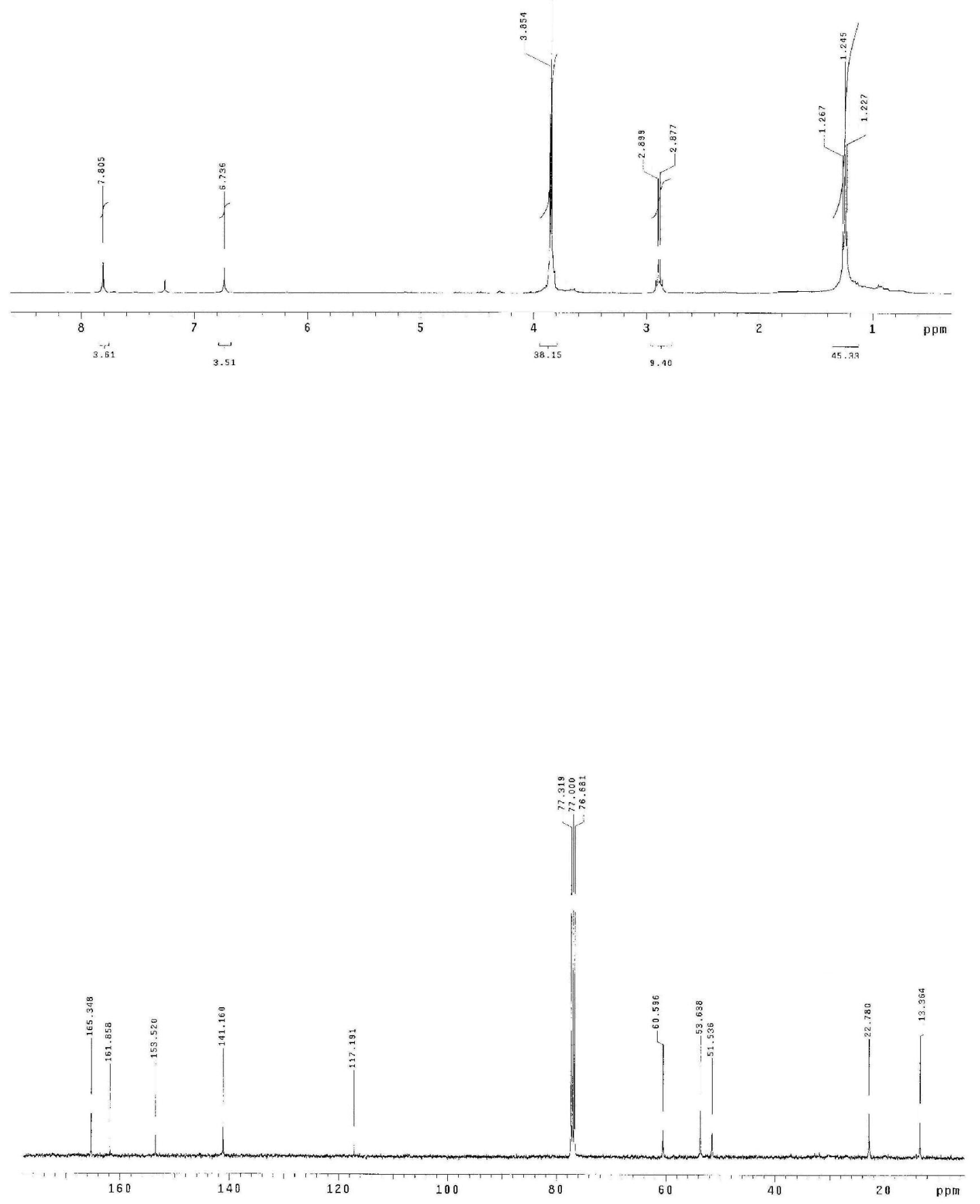


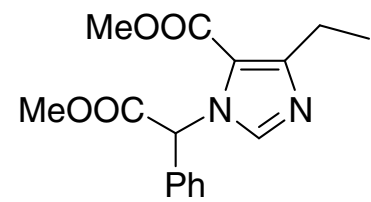

4k
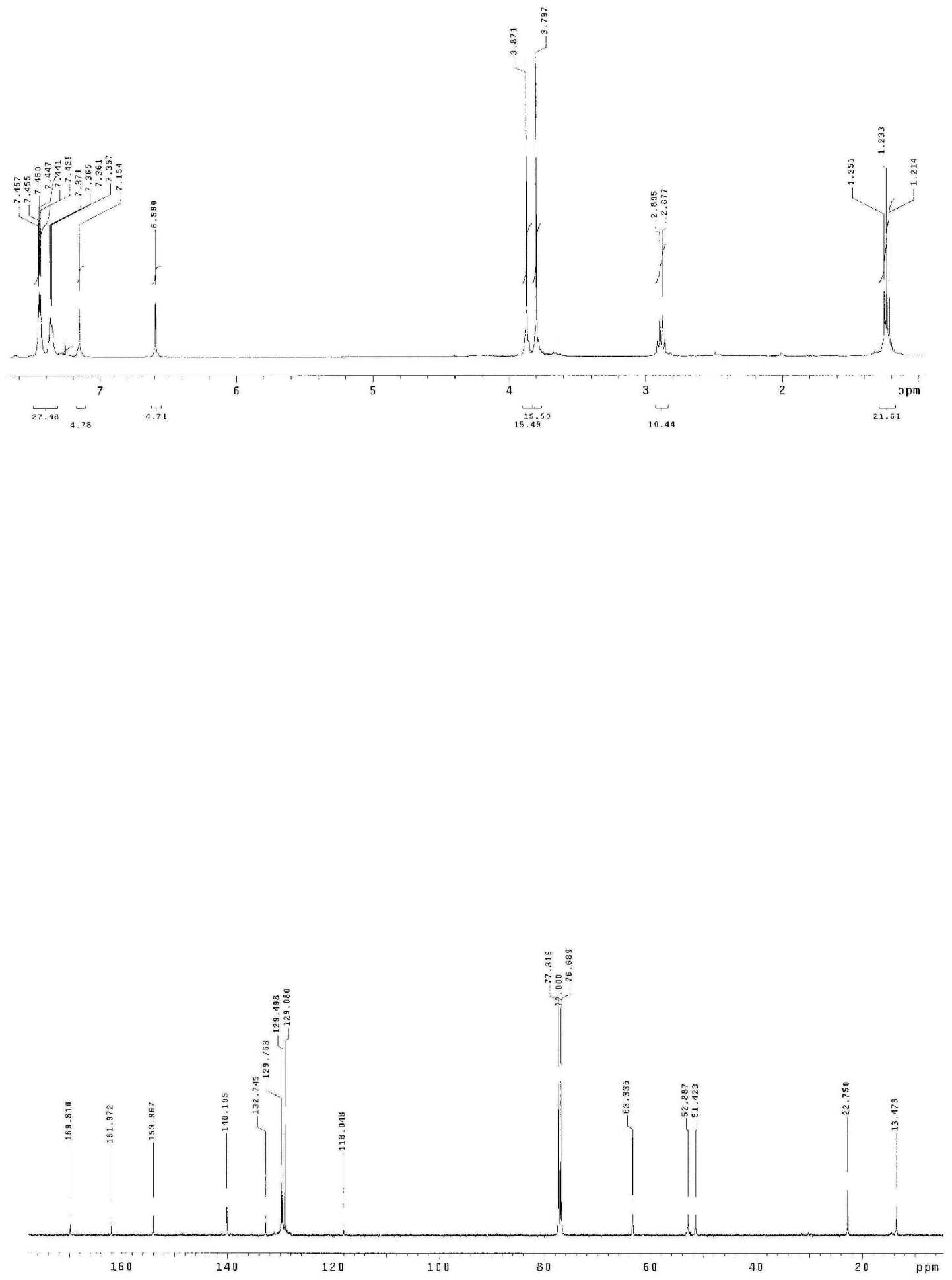


\section{References and notes}

(1) (a) Attanasi, O. A.; Filippone, P.; Mei, A.; Santeusanio, S. Synthesis 1984, 671-672. (b) Attanasi, O. A.; Filippone, P.; Mei, A.; Santeusanio, S. Synthesis 1984, 873-874.

(2) Sommer, S. Tetrahedron Lett. 1977, 18, 117-120.

(3) Davoli, P.; Forni, A.; Moretti, I.; Prati, F.; Torre, G. Tetrahedron 2001, 57, 1801-1812.

(4) (a) Antolini, L.; Forni, A.; Moretti, I.; Schenetti, L.; Prati, F. J. Chem. Soc., Perkin Trans. 2 1992, 1541-1544. (b) Prosyanik, A. V.; Bondarenko, S. V.; Loban', S. V.; Markov, V. I. Khim. Geterotsikl. Soedin. 1985, 3, 346-351.

(5) Thijs, L.; Smeets, F. L. M.; Cillissen, P. J. M.; Harmsen, J.; Zwanenburg, B. Tetrahedron 1980, $36,2141-2143$.

(6) Methyl phenylglyoxylate (2e) was prepared by standard Fischer esterification of commercially available phenylglyoxylic acid (2d), cfr. Claisen, L. Ber. 1879, 12, 626-632.

(7) Hudlicky, M. J. Org. Chem. 1980, 45, 5377-5378.

(8) Li, H.; Wang, B.; Deng, L. J. Am. Chem. Soc. 2006, 128, 732-733. 